\title{
Expedient Approach to Chiral Cyclobutanones: Asymmetric Synthesis of Cyclobut-G
}

Benjamin Darses, Andrew E. Greene, Susannah C. Coote, and Jean-François Poisson*

Département de Chimie Moléculaire (SERCO)

UMR-5250, ICMG FR-2607, CNRS

Université Joseph Fourier

BP 53, 38041 Grenoble Cedex 9, France

jean-francois.poisson@ujf-grenoble.fr

\section{SUPPORTING INFORMATION}

(2R,3R)-2-Methyl-3-((S)-1-(2,4,6-triisopropylphenyl)ethoxy)cyclobutanone (3a) S2

(2R,3R)-2-Benzyl-3-((S)-1-(2,4,6-triisopropylphenyl)ethoxy)cyclobutanone (3b) S3

(2S,3S)-2-Allyl-3-((R)-1-(2,4,6-triisopropylphenyl)ethoxy)cyclobutanone (3c) S3

(2R,3R)-2-(3-Phenylpropyl)-3-((S)-1-(2,4,6-triisopropylphenyl)ethoxy)cyclobutanone

4-((1S,4S)-2-Oxo-4-((R)-1-(2,4,6-triisopropylphenyl)ethoxy)cyclobutyl)butyl Benzoate

(2S,3S)-2-(4-(tert-Butyldimethylsilyloxy)butyl)-3-((R)-1-(2,4,6-triisopropylphenyl)ethoxy)cyclobutanone (3f)

(2S,3S)-3-((R)-1-(2,4,6-Triisopropylphenyl)ethoxy)-2-((triisopropylsilyloxy)methyl)-

cyclobutanone $(3 g)$

(S)-2-Methylene-3-((R)-1-(2,4,6-triisopropylphenyl)ethoxy)cyclobutanone (3i)

(1S,2S,3S)-3-((R)-1-(2,4,6-Triisopropylphenyl)ethoxy)-2-((triisopropylsilyloxy)methyl)cyclobutanecarbaldehyde (4)

((1S,2S,3S)-3-(1-(2,4,6-Triisopropylphenyl)ethoxy)-2-((triisopropylsilyloxy)methyl)cyclobutyl)methanol

((1S,2S,3S)-3-(1-(2,4,6-Triisopropylphenyl)ethoxy)cyclobutane-1,2-diyl)dimethanol

((1S,2S,3S)-3-(1-(2,4,6-Triisopropylphenyl)ethoxy)cyclobutyl)-1,2-dimethyl 1,2-

Dibenzoate (5)

((1S,2S,3S)-3-Hydroxycyclobutane-1,2-diyl)bis(methylene) Dibenzoate

9-((1R,2R,3S)-2,3-bis(Hydroxymethyl)cyclobutyl)-2-amino-1H-purin-6(9H)-one (7)

S8

${ }^{1} \mathrm{H}$ and ${ }^{13} \mathrm{C}$ spectra

S9-S36 
Reactions were generally carried out under argon in oven-dried glassware. Standard inert atmosphere techniques were used in handling all air and moisture sensitive reagents. Dry THF was obtained by filtration through activated molecular sieves and dry $\mathrm{CH}_{2} \mathrm{Cl}_{2}$ by filtration through activated aluminium oxide. Thin-layer chromatography was performed on $(0.2 \mathrm{~mm})$ silica sheets, which were visualized under ultraviolet light and by heating the plate after treatment with phosphomolybdic acid in ethanol, a $p$-anisaldehyde staining solution $(80 \mathrm{~mL}$ of $95 \%$ ethanol, $2.9 \mathrm{~mL}$ of sulfuric acid, $0.86 \mathrm{~mL}$ of acetic acid, $2.1 \mathrm{~mL}$ of $p$-anisaldehyde), ninhydrin in ethanol, ceric ammonium molybdate in ethanol, or basic, aqueous $\mathrm{KMnO}_{4}$. Silica gel (0.040-0.063 mm) was employed for flash column chromatography. A Fourier transform infrared spectrometer was used to record IR spectra. ${ }^{1} \mathrm{H}$ NMR and ${ }^{13} \mathrm{C}$ NMR spectra were recorded on either an $\mathrm{AV} 300,400$, or $500 \mathrm{MHz}$ apparatus. All shifts for ${ }^{1} \mathrm{H}$ spectra were referenced to the residual solvent peak and are reported in ppm. When ambiguous, proton and carbon assignments were established through COSY, HMQC, and/or DEPT experiments. Mass spectra were recorded using either DCI (ammonia/isobutane 63/37), EI, or ESI techniques. HRMS were recorded on an Orbitrap apparatus (ESI). Microanalyses were performed by the microanalysis service of the DCM.

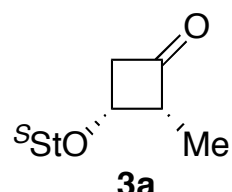

(2R,3R)-2-Methyl-3-((S)-1-(2,4,6-triisopropylphenyl)ethoxy)cyclobutanone (3a): $\mathrm{mp} 51$ ${ }^{\circ} \mathrm{C} ;[\alpha]_{\mathrm{D}}{ }^{20}-103\left(c\right.$ 1.0, $\left.\mathrm{CHCl}_{3}\right) ;{ }^{1} \mathrm{H}$ NMR $\left(400 \mathrm{MHz}, \mathrm{CDCl}_{3}\right) \delta 7.06$ (s, 1H), $6.96(\mathrm{~s}, 1 \mathrm{H}), 5.05$ $(\mathrm{q}, J=6.8 \mathrm{~Hz}, 1 \mathrm{H}), 4.18-4.11(\mathrm{~m}, 1 \mathrm{H}), 3.95$ (sept, $J=6.8 \mathrm{~Hz}, 1 \mathrm{H}), 3.34-3.23(\mathrm{~m}, 1 \mathrm{H}), 3.14$ (sept, $J=6.8 \mathrm{~Hz}, 1 \mathrm{H}), 3.11$ (ddd, $J=17.3,5.7,4.7 \mathrm{~Hz}, 1 \mathrm{H}), 2.97$ (ddd, $J=17.3,3.1,1.7 \mathrm{~Hz}$, $1 \mathrm{H}), 2.86$ (sept, $J=7.0 \mathrm{~Hz}, 1 \mathrm{H}), 1.55$ (d, $J=6.8 \mathrm{~Hz}, 3 \mathrm{H}), 1.28-1.14(\mathrm{~m}, 21 \mathrm{H}) ;{ }^{13} \mathrm{C}$ NMR $(100$ $\left.\mathrm{MHz}, \mathrm{CDCl}_{3}\right) \delta 210.6,148.8,147.6,146.0,132.3,123.3,120.5,70.8,63.9,58.1,52.0,34.0$, 29.1, 28.1, 25.1, 24.9, 24.5, 24.3, 23.9, 23.9, 23.1, 7.3; IR 2963, 2927, 2869, 1788, 1607, 1455, 1383, 1177, $1076 \mathrm{~cm}^{-1}$; MS (m/z, ESI) $337.1(\mathrm{M}+\mathrm{Li})^{+}$; Anal calcd for $\mathrm{C}_{22} \mathrm{H}_{34} \mathrm{O}_{2}: \mathrm{C}$, 79.95; H, 10.37. Found: C, 79.65; H, 10.43. 


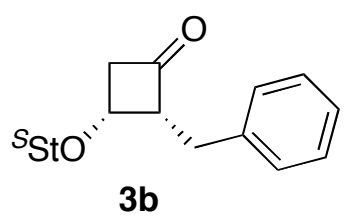

(2R,3R)-2-Benzyl-3-((S)-1-(2,4,6-triisopropylphenyl)ethoxy)cyclobutanone (3b): mp 146 ${ }^{\circ} \mathrm{C} ;[\alpha]_{\mathrm{D}}{ }^{20}-68.1\left(c\right.$ 1.0, $\left.\mathrm{CHCl}_{3}\right) ;{ }^{1} \mathrm{H}$ NMR $\left(300 \mathrm{MHz}, \mathrm{CDCl}_{3}\right) \delta$ 7.33-7.13 (m, 5H), $7.04(\mathrm{~s}$, $1 \mathrm{H}), 6.95$ (s, 1H), 5.12 (q, $J=6.8 \mathrm{~Hz}, 1 \mathrm{H}), 4.27-4.19(\mathrm{~m}, 2 \mathrm{H}), 3.86$ (sept, $J=6.7 \mathrm{~Hz}, 1 \mathrm{H})$, 3.54-3.42 (m, 1H), 3.21-3.00 (m, 5H), 2.85 (sept, $J=6.9 \mathrm{~Hz}, 1 \mathrm{H}), 1.59$ (d, $J=6.8 \mathrm{~Hz}, 3 \mathrm{H})$, 1.30-1.15 (m, 15H), 1.02 (d, $J=6.7 \mathrm{~Hz}, 3 \mathrm{H}) ;{ }^{13} \mathrm{C} \mathrm{NMR}\left(75 \mathrm{MHz}, \mathrm{CDCl}_{3}\right) \delta 208.4,148.8$, $147.7,146.0,139.9,132.0,129.0,128.3,126.0,123.3,120.6,71.2,64.5,64.1,52.2,34.0$, 29.9, 29.1, 28.1, 24.9, 24.8, 24.5, 24.4, 23.9, 23.9, 23.1; IR 2955, 2927, 2865, 1784, 1455, $1080 \mathrm{~cm}^{-1}$; MS (m/z, ESI) $413.1(\mathrm{M}+\mathrm{Li})^{+}$; Anal calcd for $\mathrm{C}_{28} \mathrm{H}_{38} \mathrm{O}_{2}: \mathrm{C}, 82.72 ; \mathrm{H}, 9.42$. Found: C, 82.65; H, 9.39.

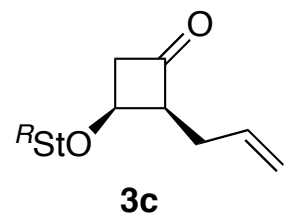

(2S,3S)-2-Allyl-3-((R)-1-(2,4,6-triisopropylphenyl)ethoxy)cyclobutanone $\quad(3 \mathrm{c}): \quad[\alpha]_{\mathrm{D}}{ }^{20}$ +75.4 (c 1.0, $\left.\mathrm{CHCl}_{3}\right) ;{ }^{1} \mathrm{H}$ NMR $\left(400 \mathrm{MHz}, \mathrm{CDCl}_{3}\right) \delta 7.06$ (s, 1H), 6.95 (s, 1H), 5.92 (ddt, $J=$ 17.0, 10.1, $6.9 \mathrm{~Hz}, 1 \mathrm{H}), 5.10-5.00(\mathrm{~m}, 3 \mathrm{H}), 4.22-4.17$ (m, 1H), 3.93 (sept, $J=6.8 \mathrm{~Hz}, 1 \mathrm{H}$ ), 3.29-3.22 (m, 1H), 3.10-3.00 (m, 3H), 2.86 (sept, $J=6.9 \mathrm{~Hz}, 1 \mathrm{H}), 2.55-2.40(\mathrm{~m}, 2 \mathrm{H}), 1.55(\mathrm{~d}$, $J=6.7 \mathrm{~Hz}, 3 \mathrm{H}), 1.26-1.16(\mathrm{~m}, 18 \mathrm{H}) ;{ }^{13} \mathrm{C} \mathrm{NMR}\left(100 \mathrm{MHz}, \mathrm{CDCl}_{3}\right) \delta$ 208.9, 148.8, 147.6, 146.0, 136.0, 132.1, 123.3, 120.6, 115.9, 71.0, 63.8, 62.7, 52.3, 34.0, 29.0, 28.3, 28.1, 25.2, 25.0, 24.5, 24.4, 23.90, 23.88, 23.1; IR 3075, 2959, 2927, 2865, 1784, 1604, 1173, 1105, $1076 \mathrm{~cm}^{-1}$; MS $\left(\mathrm{m} / z\right.$, ESI) $363.1(\mathrm{M}+\mathrm{Li})^{+}$; HRMS-ESI calcd for $\mathrm{C}_{24} \mathrm{H}_{36} \mathrm{O}_{3} \mathrm{Na}(\mathrm{M}+\mathrm{Na})^{+}$: 395.2557. Found: 395.2565.

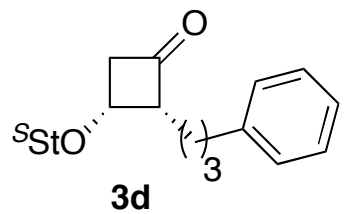

(2R,3R)-2-(3-Phenylpropyl)-3-((S)-1-(2,4,6-triisopropylphenyl)ethoxy)cyclobutanone (3d): $(\mathrm{dr}=96: 4) ;{ }^{1} \mathrm{H}$ NMR $\left(300 \mathrm{MHz}, \mathrm{CDCl}_{3}\right) \delta$ 7.30-7.11 (m, 5H), $7.05(\mathrm{~s}, 1 \mathrm{H}), 6.95(\mathrm{~s}, 1 \mathrm{H})$, $5.05(\mathrm{q}, J=6.8 \mathrm{~Hz}, 1 \mathrm{H}), 4.20-4.11(\mathrm{~m}, 1 \mathrm{H}), 3.89$ (sept, $J=6.8 \mathrm{~Hz}, 1 \mathrm{H}), 3.22-3.09(\mathrm{~m}, 2 \mathrm{H})$, 
3.09-2.94 (m, 2H), $2.86(\mathrm{sept}, J=6.9 \mathrm{~Hz}, 1 \mathrm{H}), 2.69-2.52(\mathrm{~m}, 2 \mathrm{H}), 1.90-1.64(\mathrm{~m}, 4 \mathrm{H}), 1.53(\mathrm{~d}$, $J=6.8 \mathrm{~Hz}, 3 \mathrm{H}), 1.28-1.06(\mathrm{~m}, 18 \mathrm{H}) ;{ }^{13} \mathrm{C} \mathrm{NMR}\left(75 \mathrm{MHz}, \mathrm{CDCl}_{3}\right) \delta 209.8,148.8,147.6$, $146.0,142.2$, 132.2, 128.4, 128.2, 125.6, 123.2, 120.5, 71.0, 64.0, 63.2, 52.3, 35.9, 34.0, 29.5, 29.1, 28.1, 25.1, 24.9, 24.5, 24.4, 23.91, 23.89, 23.6, 23.1; IR 3024, 2959, 2927, 2865, 1781, $1457,1080 \mathrm{~cm}^{-1}$; MS (m/z, ESI) $441.1(\mathrm{M}+\mathrm{Li})^{+}$; Anal calcd for $\mathrm{C}_{30} \mathrm{H}_{42} \mathrm{O}_{2}: \mathrm{C}, 82.9 ; \mathrm{H}, 9.74$. Found: C, 83.24; H, 9.85.

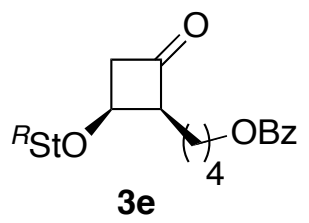

4-((1S,4S)-2-Oxo-4-((R)-1-(2,4,6-triisopropylphenyl)ethoxy)cyclobutyl)butyl Benzoate (3e): $[\alpha]_{\mathrm{D}}{ }^{20}+57.5\left(c 1.0, \mathrm{CHCl}_{3}\right) ;{ }^{1} \mathrm{H}$ NMR $\left(300 \mathrm{MHz}, \mathrm{CDCl}_{3}\right) \delta 8.08-8.00(\mathrm{~m}, 2 \mathrm{H}), 7.58-7.50$ $(\mathrm{m}, 1 \mathrm{H}), 7.47-7.38(\mathrm{~m}, 2 \mathrm{H}), 7.05(\mathrm{~s}, 1 \mathrm{H}), 6.95(\mathrm{~s}, 1 \mathrm{H}), 5,06$ (q, $J=6.8 \mathrm{~Hz}, 1 \mathrm{H}), 4.39-4.22(\mathrm{~m}$, 2H), 4.21-4.13 (m, 1H), 3.89 (sept, $J=6.7 \mathrm{~Hz}, 1 \mathrm{H}), 3.26-3.10$ (m, 2H), 3.10-2.97 (m, 2H), 2.86 (sept, $J=6.9 \mathrm{~Hz}, 1 \mathrm{H}), 1.91-1.60(\mathrm{~m}, 6 \mathrm{H}), 1.54(\mathrm{~d}, J=6.8 \mathrm{~Hz}, 3 \mathrm{H}), 1.28-1.14(\mathrm{~m}, 18 \mathrm{H})$;

${ }^{13} \mathrm{C}$ NMR $\left(75 \mathrm{MHz}, \mathrm{CDCl}_{3}\right) \delta$ 209.6, 166.6, 148.7, 147.6, 146.0, 132.7, 132.1, 129.5, 128.3, $123.2,120.6,70.9,64.8,63.9,63.1,52.2,33.9,29.0,28.8,28.1,25.1,24.9,24.8,24.5,24.4$, 23.9, 23.6, 23.1; IR 2959, 2927, 2865, 1784, 1719, 1451, 1116, $1069 \mathrm{~cm}^{-1}$; MS (m/z, ESI) $499.1(\mathrm{M}+\mathrm{Li})^{+}$; Anal calcd for $\mathrm{C}_{32} \mathrm{H}_{44} \mathrm{O}_{4}$ : C, 78.01; H, 9.01. Found: C, 78.01; H, 8.96.

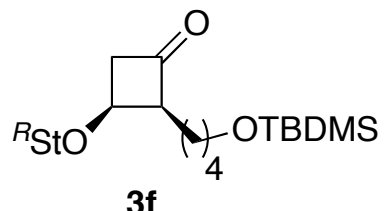

(2S,3S)-2-(4-(tert-Butyldimethylsilyloxy)butyl)-3-((R)-1-(2,4,6-triisopropylphenyl)ethoxy)cyclobutanone (3f): $[\alpha]_{\mathrm{D}}{ }^{20}+56.3\left(c 1.0, \mathrm{CHCl}_{3}\right) ;{ }^{1} \mathrm{H} \mathrm{NMR}\left(400 \mathrm{MHz}, \mathrm{CDCl}_{3}\right) \delta 7.06$ (s, 1H), $6.95(\mathrm{~s}, 1 \mathrm{H}), 5.05(\mathrm{q}, J=6.8 \mathrm{~Hz}, 1 \mathrm{H}), 4.19-4.12(\mathrm{~m}, 1 \mathrm{H}), 3.93$ (sept, $J=6.8 \mathrm{~Hz}, 1 \mathrm{H})$, 3.59 (t, $J=6.3 \mathrm{~Hz}, 2 \mathrm{H}), 3.19-3.08(\mathrm{~m}, 2 \mathrm{H}), 3.08-2.97(\mathrm{~m}, 2 \mathrm{H}), 2.86$ (sept, $J=6.9 \mathrm{~Hz}, 1 \mathrm{H})$, $1.82-1.62(\mathrm{~m}, 2 \mathrm{H}), 1.61-1.46(\mathrm{~m}, 6 \mathrm{H}), 1.46-1.33(\mathrm{~m}, 1 \mathrm{H}), 1.30-1.14(\mathrm{~m}, 18 \mathrm{H}), 0.89(\mathrm{~s}, 9 \mathrm{H})$, 0.04 (s, 6H); ${ }^{13} \mathrm{C} \mathrm{NMR}\left(100 \mathrm{MHz}, \mathrm{CDCl}_{3}\right) \delta$ 210.0, 148.8, 147.6, 146.0, 132.2, 123.3, 120.6, $70.9,63.9,63.4,63.0,52.2,34.0,32.9,29.0,28.1,26.0,25.1,25.0,24.5,24.4,24.2,23.92$, 23.90, 23.8, 23.1, 18.3, -5.3; IR 2955, 2923, 2858, 1788, 1607, 1463, 1257, $1101 \mathrm{~cm}^{-1}$; MS 
$\left(\mathrm{m} / z\right.$, ESI) $509.3(\mathrm{M}+\mathrm{Li})^{+}$; HRMS-ESI calcd for $\mathrm{C}_{31} \mathrm{H}_{54} \mathrm{O}_{3} \mathrm{SiNa}(\mathrm{M}+\mathrm{Na})^{+}:$525.3634. Found: 525.3741 .

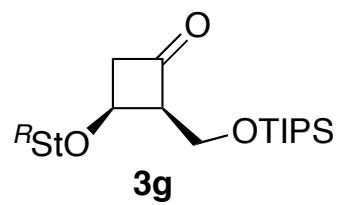

(2S,3S)-3-((R)-1-(2,4,6-Triisopropylphenyl)ethoxy)-2-((triisopropylsilyloxy)methyl)cyclobutanone (3g): $[\alpha]_{\mathrm{D}}{ }^{20}+57.2\left(c 1.0, \mathrm{CHCl}_{3}\right) ;{ }^{1} \mathrm{H}$ NMR $\left(300 \mathrm{MHz}, \mathrm{CDCl}_{3}\right) \delta 7.05(\mathrm{~s}, 1 \mathrm{H})$, 6.95 (s, 1H), 5.07 (q, $J=6.8 \mathrm{~Hz}, 1 \mathrm{H}), 4.24-4.15(\mathrm{~m}, 1 \mathrm{H}), 4.14-4.03(\mathrm{~m}, 2 \mathrm{H}), 3.96$ (sept, $J=$ $6.7 \mathrm{~Hz}, 1 \mathrm{H}), 3.35-3.25(\mathrm{~m}, 1 \mathrm{H}), 3.22-2.99$ (m, 3H), 2.86 (sept, $J=6.9 \mathrm{~Hz}, 1 \mathrm{H}), 1.55$ (d, $J=$ $6.8 \mathrm{~Hz}, 3 \mathrm{H}), 1.29-1.00(\mathrm{~m}, 39 \mathrm{H}) ;{ }^{13} \mathrm{C} \mathrm{NMR}\left(75 \mathrm{MHz}, \mathrm{CDCl}_{3}\right) \delta$ 207.0, 148.7, 147.6, 146.0, $132.4,123.3,120.5,71.2,65.8,63.4,57.8,54.2,34.0,29.0,28.2,25.1,24.7,24.5,23.91$, 23.89, 23.1, 18.0, 11.9; IR 2962, 2890, 2869, 1789, 1462, 1383, 1122, 1098, 1076, $880 \mathrm{~cm}^{-1}$; MS (m/z, ESI) $509.5(\mathrm{M}+\mathrm{Li})^{+}$; Anal calcd for $\mathrm{C}_{31} \mathrm{H}_{54} \mathrm{O}_{3} \mathrm{Si}: \mathrm{C}, 74.05 ; \mathrm{H}, 10.83$. Found: $\mathrm{C}$, 73.98; H, 10.97.

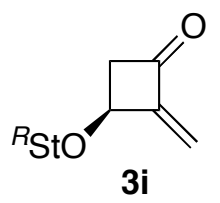

(S)-2-Methylene-3-((R)-1-(2,4,6-triisopropylphenyl)ethoxy)cyclobutanone $\quad(3 i): \quad$ two diastereoisomers $(\mathrm{dr}=92: 8)$; Data for the major diastereoisomer: ${ }^{1} \mathrm{H}$ NMR $(400 \mathrm{MHz}$, $\left.\mathrm{CDCl}_{3}\right) \delta 7.14-6.90(\mathrm{~m}, 2 \mathrm{H}), 5.83(\mathrm{~d}, J=2.2 \mathrm{~Hz}, 1 \mathrm{H}), 5.20(\mathrm{~d}, J=1.0 \mathrm{~Hz}, 1 \mathrm{H}), 5.12(\mathrm{q}, J=$ $6.8 \mathrm{~Hz}, 1 \mathrm{H}), 4.61-4.56(\mathrm{~m}, 1 \mathrm{H}), 3.97(\mathrm{~m}, 1 \mathrm{H}), 3.16(\mathrm{~m}, 1 \mathrm{H}), 3.10(\mathrm{dd}, J=17.4,6.4 \mathrm{~Hz}, 1 \mathrm{H})$, $3.00(\mathrm{dd}, J=17.4,4.8 \mathrm{~Hz}, 1 \mathrm{H}), 2.87$ (sept, $J=6.9 \mathrm{~Hz}, 1 \mathrm{H}), 1.61$ (d, $J=6.8 \mathrm{~Hz}, 3 \mathrm{H}), 1.29$ $1.19(\mathrm{~m}, 18 \mathrm{H}) ;{ }^{13} \mathrm{C} \mathrm{NMR}\left(75 \mathrm{MHz}, \mathrm{CDCl}_{3}\right) \delta$ 196.4, 156.2, 147.9, 132.0, 123.3, 120.7, 115.5, 72.1, 68.2, 52.9, 34.0, 29.0, 28.3, 24.7, 24.6, 23.91, 23.88, 23.1; IR 2955, 2927, 2865, 1766, $1080 \mathrm{~cm}^{-1}$; MS (m/z, ESI) $335.1(\mathrm{M}+\mathrm{Li})^{+}$; HRMS-ESI calcd for $\mathrm{C}_{22} \mathrm{H}_{32} \mathrm{O}_{2} \mathrm{Na}(\mathrm{M}+\mathrm{Na})^{+}$: 351.2294. Found: 351.2303. 


\section{Cyclobut-G synthesis:}

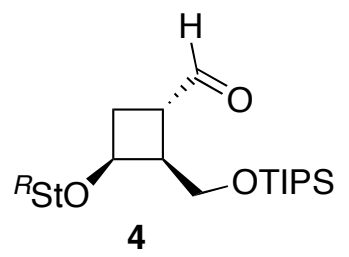

(1S,2S,3S)-3-((R)-1-(2,4,6-Triisopropylphenyl)ethoxy)-2-((triisopropylsilyloxy)methyl)cyclobutanecarbaldehyde (4): $[\alpha]_{\mathrm{D}}{ }^{20}+68.4\left(c 1.0, \mathrm{CHCl}_{3}\right) ;{ }^{1} \mathrm{H} \mathrm{NMR}\left(300 \mathrm{MHz}, \mathrm{CDCl}_{3}\right) \delta$ 9.77 (d, $J=1.5 \mathrm{~Hz}, 1 \mathrm{H}), 7.01(\mathrm{~s}, 1 \mathrm{H}), 6.92(\mathrm{~s}, 1 \mathrm{H}), 4.91$ (q, $J=6.8 \mathrm{~Hz}, 1 \mathrm{H}), 4.05-3.74(\mathrm{~m}$, 4H), 3.24-3.15 (m, 1H), 3.15-3.00 (m, 1H), 2.84 (sept, $J=6.9 \mathrm{~Hz}, 1 \mathrm{H}), 2.79-2.69$ (m, 1H), 2.58-2.47 (m, 1H), 2.27-2.15 (m, 1H), $1.48(\mathrm{~d}, J=6.8 \mathrm{~Hz}, 3 \mathrm{H}), 1.28-1.00(\mathrm{~m}, 39 \mathrm{H}) ;{ }^{13} \mathrm{C} \mathrm{NMR}$ $\left(75 \mathrm{MHz}, \mathrm{CDCl}_{3}\right) \delta 202.2,148.5,147.3,145.6,133.1,123.1,120.4,70.4,69.3,67.6,61.4$, $44.4,44.1,40.2,33.9,29.0,28.3,28.2,24.5,23.9,23.8,23.2$, , 18.00, , 12.0; IR 2954, 2892, 2862, 1719, 1466, 1116, $1073 \mathrm{~cm}^{-1}$; MS (m/z, ESI) $523.3(\mathrm{M}+\mathrm{Li})^{+}$; Anal calcd for $\mathrm{C}_{32} \mathrm{H}_{56} \mathrm{O}_{3} \mathrm{Si}: \mathrm{C}, 74.37 ; \mathrm{H}, 10.92$. Found: C, 14.28; H, 11.03.

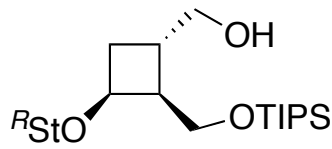

((1S,2S,3S)-3-(1-(2,4,6-Triisopropylphenyl)ethoxy)-2-((triisopropylsilyloxy)methyl)cyclobutyl)methanol: $[\alpha]_{\mathrm{D}}{ }^{20}+37.6\left(c 1.0, \mathrm{CHCl}_{3}\right) ;{ }^{1} \mathrm{H} \mathrm{NMR}\left(300 \mathrm{MHz}, \mathrm{CDCl}_{3}\right) \delta 7.02(\mathrm{~s}$, $1 \mathrm{H}), 6.91(\mathrm{~s}, 1 \mathrm{H}), 4.96(\mathrm{q}, J=6.8 \mathrm{~Hz}, 1 \mathrm{H}), 3.97-3.77(\mathrm{~m}, 4 \mathrm{H}), 3.70-3.60(\mathrm{~m}, 1 \mathrm{H}), 3.36(\mathrm{dt}, J=$ 10.2, 1.4 Hz, 1H), 3.16-3.02 (m, 1H), 2.97 (dd, $J=9.0,1.5 \mathrm{~Hz}, 1 \mathrm{H}), 2.84$ (sept, $J=6.9 \mathrm{~Hz}$, $1 \mathrm{H}), 2.75-2.60(\mathrm{~m}, 1 \mathrm{H}), 2.30-2.19(\mathrm{~m}, 1 \mathrm{H}), 2.15-2.05(\mathrm{~m}, 1 \mathrm{H}), 1.75-1.64(\mathrm{~m}, 1 \mathrm{H}), 1.50$ (d, $J=$ $6.8 \mathrm{~Hz}, 3 \mathrm{H}), 1.27-1.00(\mathrm{~m}, 39 \mathrm{H}) ;{ }^{13} \mathrm{C} \mathrm{NMR}\left(75 \mathrm{MHz}, \mathrm{CDCl}_{3}\right) \delta 148.5,147.1,145.7,133.3$, $123.0,120.2,70.6,69.5,66.7,62.4,47.1,39.9,33.9,28.9,28.4,28.0,25.1,24.8,24.4,24.2$, 23.9, 23.8, 23.3, 17.9, 11.9; IR 3411, 2959, 2894, 2865, 1459, 1101, $1072 \mathrm{~cm}^{-1}$; MS $(\mathrm{m} / z$, ESI) $525.3(\mathrm{M}+\mathrm{Li})^{+}$; HRMS-ESI calcd for $\mathrm{C}_{32} \mathrm{H}_{58} \mathrm{O}_{3} \mathrm{SiNa}(\mathrm{M}+\mathrm{Na})^{+}$: 541.4047. Found 541.4044 .

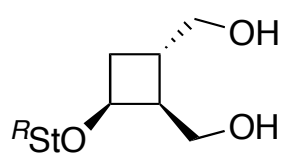

((1S,2S,3S)-3-(1-(2,4,6-Triisopropylphenyl)ethoxy)cyclobutane-1,2-diyl)dimethanol:

$[\alpha]_{\mathrm{D}}{ }^{20}+107\left(c\right.$ 1.0, $\left.\mathrm{CHCl}_{3}\right) ;{ }^{1} \mathrm{H} \mathrm{NMR}\left(300 \mathrm{MHz}, \mathrm{CDCl}_{3}\right) \delta 7.03(\mathrm{~s}, 1 \mathrm{H}), 6.93(\mathrm{~s}, 1 \mathrm{H}), 5.01$ (q, $J=6.8 \mathrm{~Hz}, 1 \mathrm{H}), 4.04(\mathrm{dt}, J=6.9,5.0 \mathrm{~Hz}, 1 \mathrm{H}), 3.90-3.74(\mathrm{~m}, 3 \mathrm{H}), 3.60(\mathrm{dd}, J=10.6,6.3 \mathrm{~Hz}$, 
1H), 3.50 (dd, $J=10.6,7.4 \mathrm{~Hz}, 1 \mathrm{H}), 3.10$ (sept, $J=6.7 \mathrm{~Hz}, 1 \mathrm{H}), 2.85$ (sept, $J=6.9 \mathrm{~Hz}, 1 \mathrm{H}$ ), 2.51-2.31 (m, 2H), 2.24-2.13 (m, 1H), 1.98-1.87 (m, 1H), 1.53 (d, $J=6.8 \mathrm{~Hz}, 3 \mathrm{H}), 1.28-1.13$ $(\mathrm{m}, 18 \mathrm{H}) ;{ }^{13} \mathrm{C} \mathrm{NMR}\left(75 \mathrm{MHz}, \mathrm{CDCl}_{3}\right) \delta$ 148.5, 147.3, 145.9, 132.3, 123.0, 120.3, 70.9, 69.6, $65.7,62.2,44.1,34.7,33.8,29.7,28.9,28.3,24.9,24.6,24.4,24.3,23.80,23.77,23.2$; IR 3364, 2959, 2930, 2869, 1611, 1463, 1383, 1112, 1080, $1029 \mathrm{~cm}^{-1}$; MS (m/z, ESI) 369.1 $(\mathrm{M}+\mathrm{Li})^{+}$; HRMS-ESI calcd for $\mathrm{C}_{23} \mathrm{H}_{38} \mathrm{O}_{3} \mathrm{Na}(\mathrm{M}+\mathrm{Na})^{+}:$285.2713. Found 285.2716.

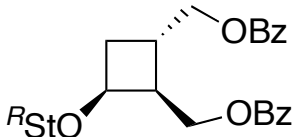

5

\section{((1S,2S,3S)-3-(1-(2,4,6-Triisopropylphenyl)ethoxy)cyclobutyl)-1,2-dimethyl}

Dibenzoate (5): $\mathrm{mp} 100{ }^{\circ} \mathrm{C}$; $[\alpha]_{\mathrm{D}}{ }^{20}+51.0\left(c\right.$ 1.0, $\left.\mathrm{CHCl}_{3}\right) ;{ }^{1} \mathrm{H} \mathrm{NMR}\left(300 \mathrm{MHz}, \mathrm{CDCl}_{3}\right) \delta 8.07-$ $7.88(\mathrm{~m}, 4 \mathrm{H}), 7.58-7.46(\mathrm{~m}, 2 \mathrm{H}), 7.42-7.27(\mathrm{~m}, 4 \mathrm{H}), 7.03(\mathrm{~s}, 1 \mathrm{H}), 6.93(\mathrm{~s}, 1 \mathrm{H}), 5.00(\mathrm{q}, J=$ $6.8 \mathrm{~Hz}, 1 \mathrm{H}), 4.71-4.61(\mathrm{~m}, 2 \mathrm{H}), 4.36$ (dd, $J=11.1,5.9 \mathrm{~Hz}, 1 \mathrm{H}), 4.28$ (dd, $J=11.1,7.2 \mathrm{~Hz}$, $1 \mathrm{H}), 4.20-4.11(\mathrm{~m}, 1 \mathrm{H}), 4.00-3.81(\mathrm{~m}, 1 \mathrm{H}), 3.23-3.02(\mathrm{~m}, 1 \mathrm{H}), 2.95-2.64(\mathrm{~m}, 3 \mathrm{H}), 2.48-2.33$ $(\mathrm{m}, 1 \mathrm{H}), 2.19-2.04(\mathrm{~m}, 1 \mathrm{H}), 1.51(\mathrm{~d}, J=6.8 \mathrm{~Hz}, 3 \mathrm{H}), 1.32-1.11(\mathrm{~m}, 18 \mathrm{H}) ;{ }^{13} \mathrm{C} \mathrm{NMR}(75 \mathrm{MHz}$, $\left.\mathrm{CDCl}_{3}\right) \delta 166.7,166.5,148.7,147.3,145.8,134.5,132.7,130.5,130.4,130.0,129.50,129.47$, $128.8,128.3,128.2,123.2,120.4,69.7,68.9,67.5,64.1,41.3,33.9,32.3,30.5,29.0,28.2$, 25.0, 24.6, 24.3, 23.90, 23.89, 23.3; IR 3060, 2963, 2930, 2865, 1792, 1719, 1604, 1455, 1275, $1119 \mathrm{~cm}^{-1}$; MS (m/z, ESI): $577.1(\mathrm{M}+\mathrm{Li})^{+}$; HRMS-ESI: calcd for $\mathrm{C}_{37} \mathrm{H}_{46} \mathrm{O}_{5} \mathrm{Na}$ $(\mathrm{M}+\mathrm{Na})^{+}:$593.3237. Found 593.3231.

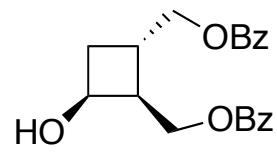

((1S,2S,3S)-3-Hydroxycyclobutane-1,2-diyl)bis(methylene) Dibenzoate: ${ }^{[16]}$ mp $73{ }^{\circ} \mathrm{C}$; $[\alpha]_{\mathrm{D}}{ }^{20}-12.1$ (c 1.0, $\left.\mathrm{CHCl}_{3}\right) ;{ }^{1} \mathrm{H}$ NMR $\left(400 \mathrm{MHz}, \mathrm{CDCl}_{3}\right) \delta$ 8.07-7.99 (m, 4H), 7.60-7.53 (m, 2H), 7.47-7.39 (m, 4H), $4.84(\mathrm{dd}, J=11.5,8.9 \mathrm{~Hz}, 1 \mathrm{H}), 4.48(\mathrm{~m}, 1 \mathrm{H}), 4.41$ (dd, $J=11.2,5.5$ $\mathrm{Hz}, 1 \mathrm{H}), 4.34(\mathrm{dd}, J=11.2,6.7 \mathrm{~Hz}, 1 \mathrm{H}), 4.27$ (dd, $J=11.5,4.5 \mathrm{~Hz}, 1 \mathrm{H}), 3.13$ (d, $J=3.0 \mathrm{~Hz}$, $1 \mathrm{H}), 2.82-2.72(\mathrm{~m}, 1 \mathrm{H}), 2.71-2.62(\mathrm{~m}, 1 \mathrm{H}), 2.25-2.10(\mathrm{~m}, 2 \mathrm{H}) ;{ }^{13} \mathrm{C} \mathrm{NMR}\left(100 \mathrm{MHz}, \mathrm{CDCl}_{3}\right)$ ठ $167.4,166.6,133.2,133.1,130.1,129.8,129.7,129.6,128.4,67.2,66.0,63.1,43.3,33.0$, 31.4; IR 3461, 2937, 1714, 1275, $1112 \mathrm{~cm}^{-1}$; MS ( $/ z / z$, ESI) $347.0(\mathrm{M}+\mathrm{Li})^{+}$; HRMS-ESI calcd for $\mathrm{C}_{20} \mathrm{H}_{20} \mathrm{O}_{5} \mathrm{Na}(\mathrm{M}+\mathrm{Na})^{+}: 363.1203$. Found 363.1210. 


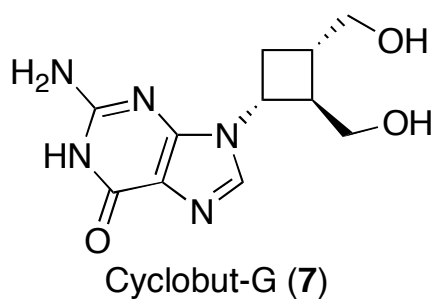

9-((1R,2R,3S)-2,3-bis(Hydroxymethyl)cyclobutyl)-2-amino-1H-purin-6(9H)-one (7): $:^{[16]}$ mp 275-280 ${ }^{\circ} \mathrm{C}(\mathrm{dec})\left[\right.$ lit. $\left.^{[16 \mathrm{~b}]} \mathrm{mp} \sim 290{ }^{\circ} \mathrm{C}(\mathrm{dec})\right]$; $[\alpha]_{\mathrm{D}}{ }^{20}-24.7$ (c 1.0, DMSO) $\left[\right.$ lit. $^{[16 \mathrm{~b}]}[\alpha]_{\mathrm{D}}{ }^{22}$ -24.4 (c 1.0, DMSO)]; ${ }^{1} \mathrm{H}$ NMR (400 MHz, DMSO) $\delta 10.52$ (br s, 1H), 7.82 (s, 1H), 6.37 (s, $1 \mathrm{H}), 4.62(\mathrm{t}, J=4.9 \mathrm{~Hz}, 1 \mathrm{H}), 4.58(\mathrm{t}, J=5.1 \mathrm{~Hz}, 1 \mathrm{H}), 4.42(\mathrm{dd}, J=16.6,8.4 \mathrm{~Hz}, 1 \mathrm{H}), 3.54-$ $3.42(\mathrm{~m}, 4 \mathrm{H}), 2.72-2.63(\mathrm{~m}, 1 \mathrm{H}), 2.40-2.29(\mathrm{~m}, 1 \mathrm{H}), 2.09-1.97(\mathrm{~m}, 2 \mathrm{H}) ;{ }^{13} \mathrm{C}$ NMR $(75 \mathrm{MHz}$, $\left.\mathrm{CDCl}_{3}\right) \delta 156.8,153.2150 .9,135.8,116.8,63.5,61.4,47.7,46.5,33.1,29.6 ; \mathrm{MS}(\mathrm{m} / \mathrm{z}, \mathrm{ESI})$ $265.9(\mathrm{M}+\mathrm{H})^{+}$. 


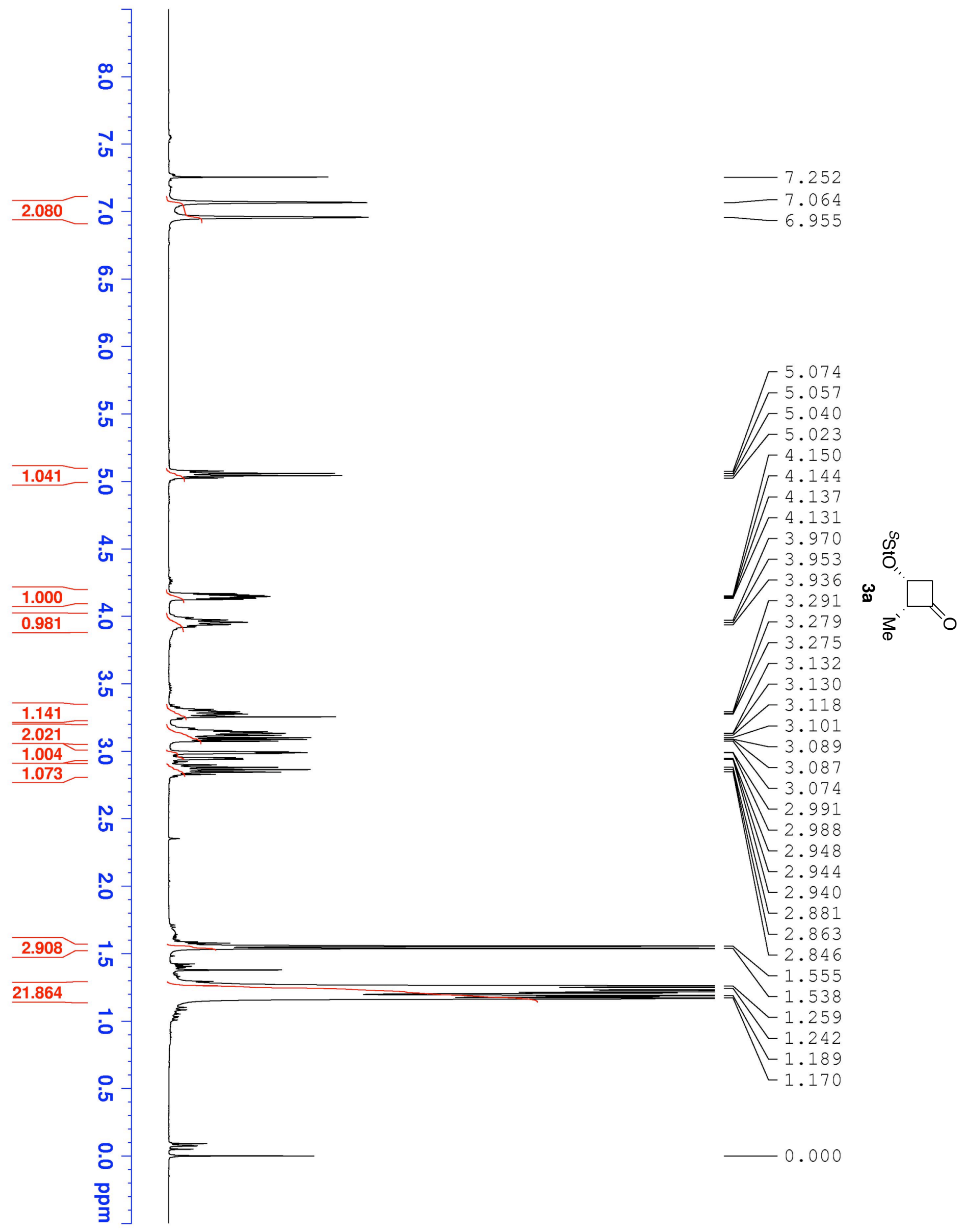




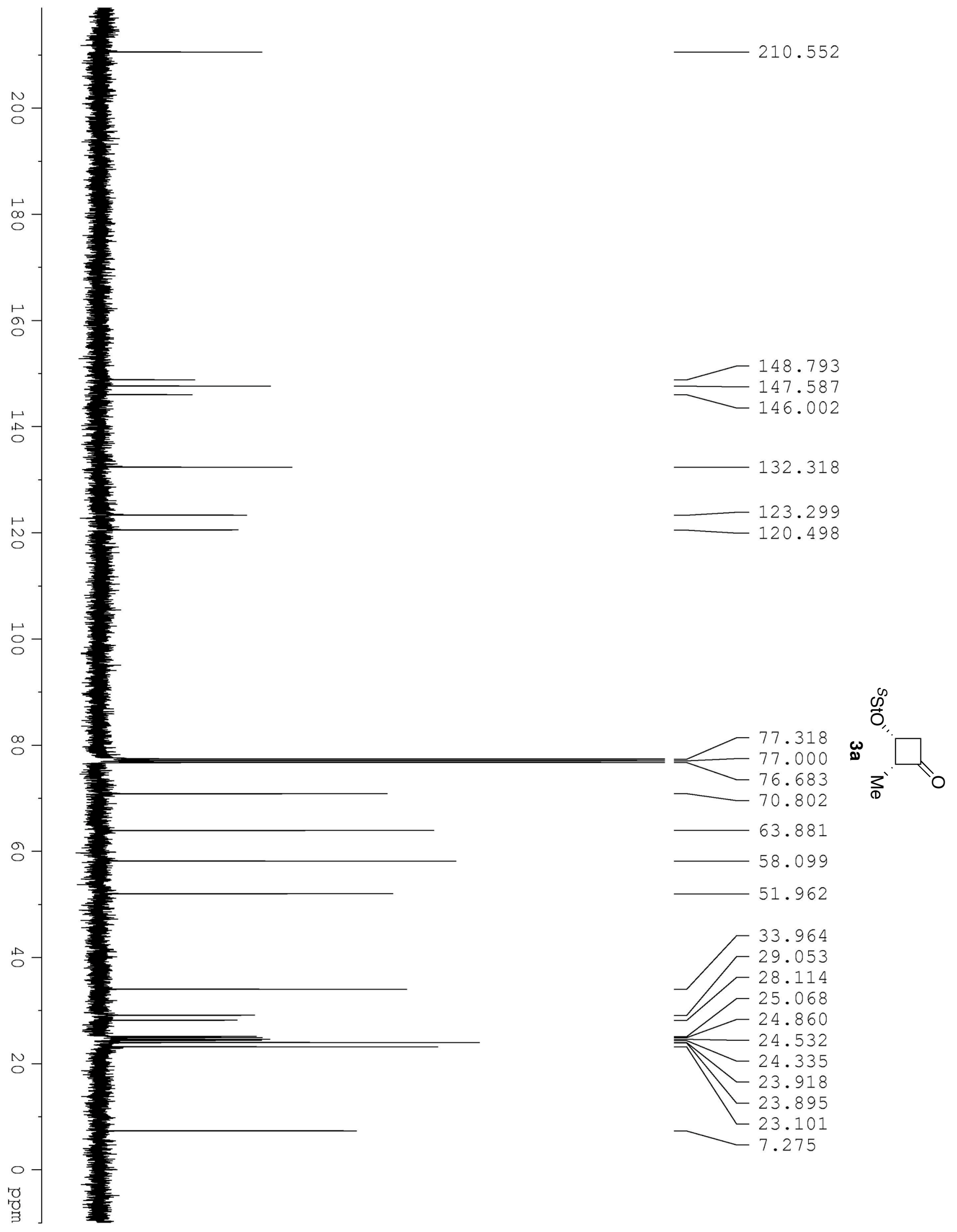




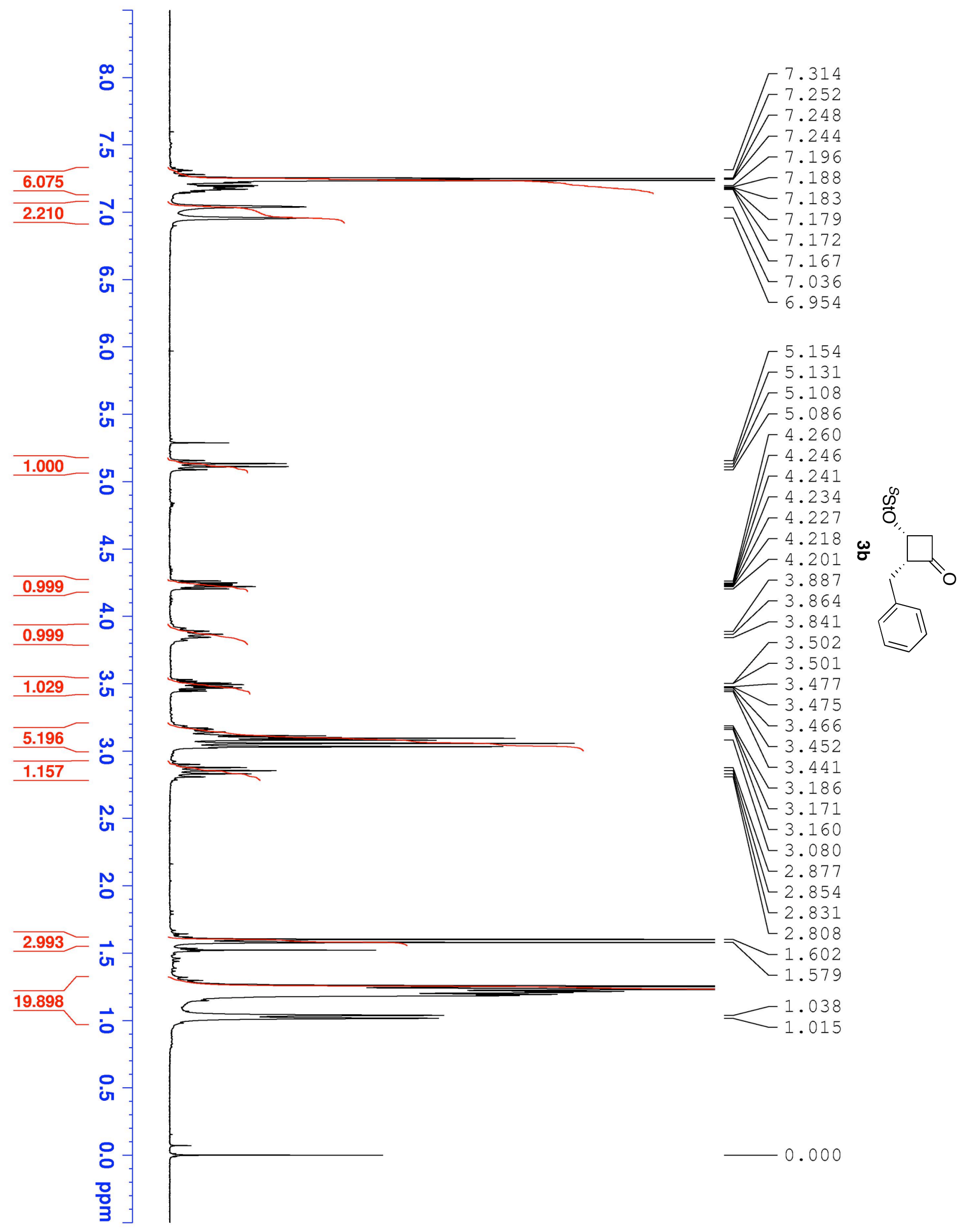




$$
\text { ba }
$$




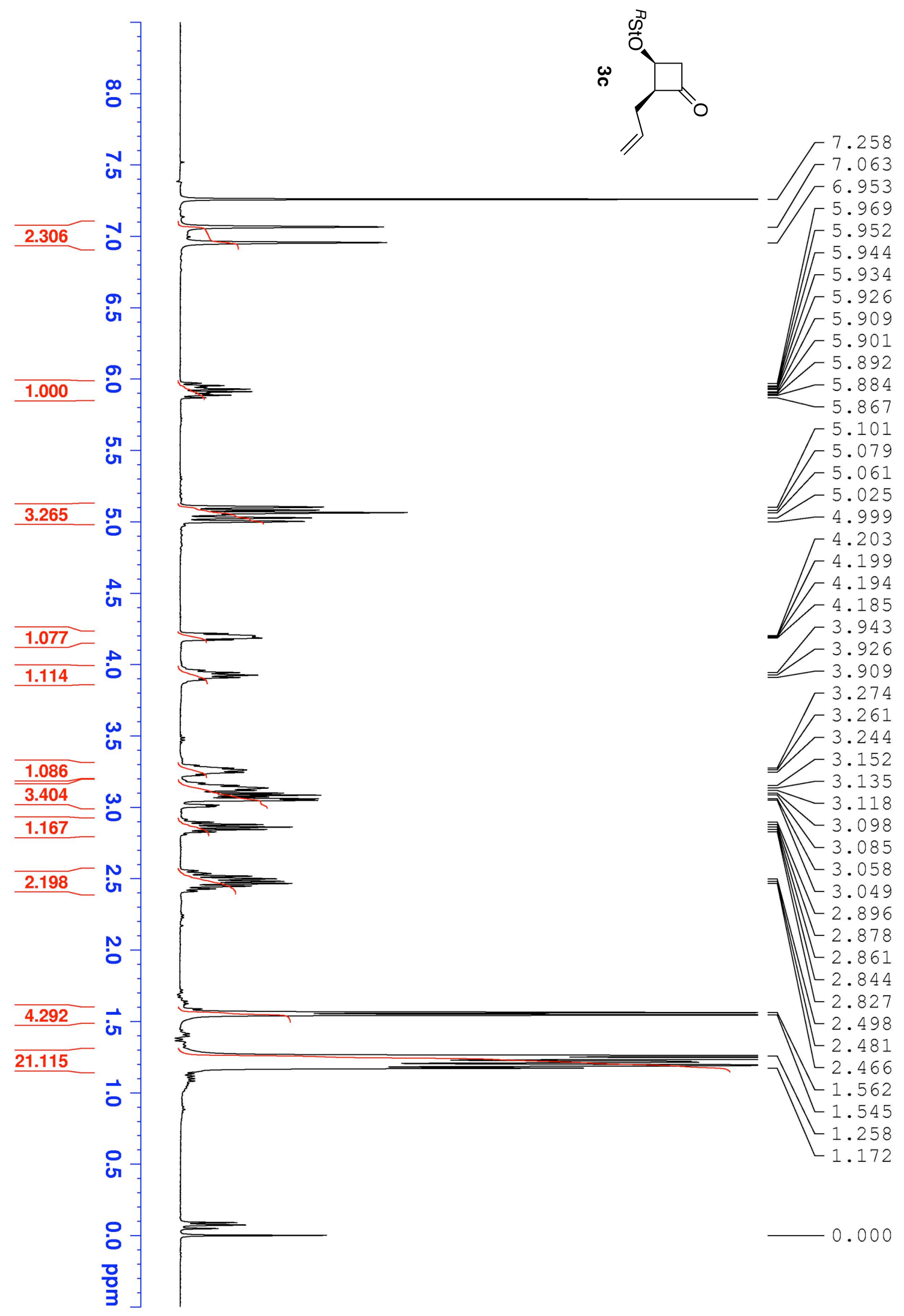




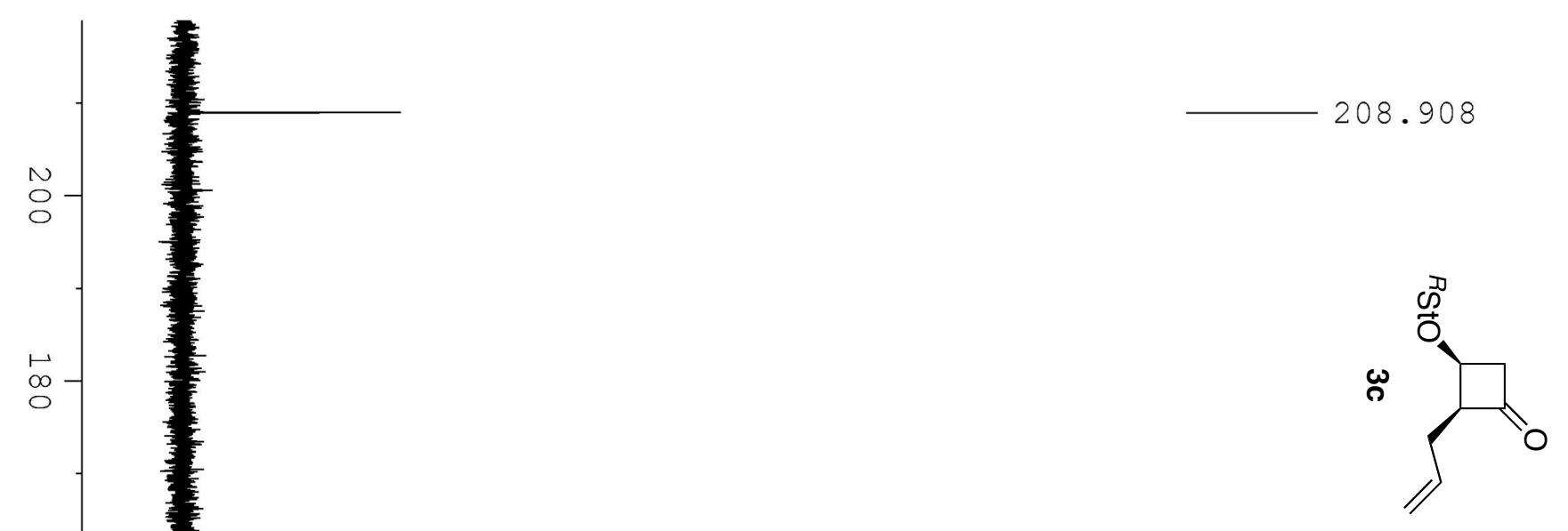

뭉

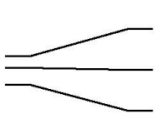

148.778
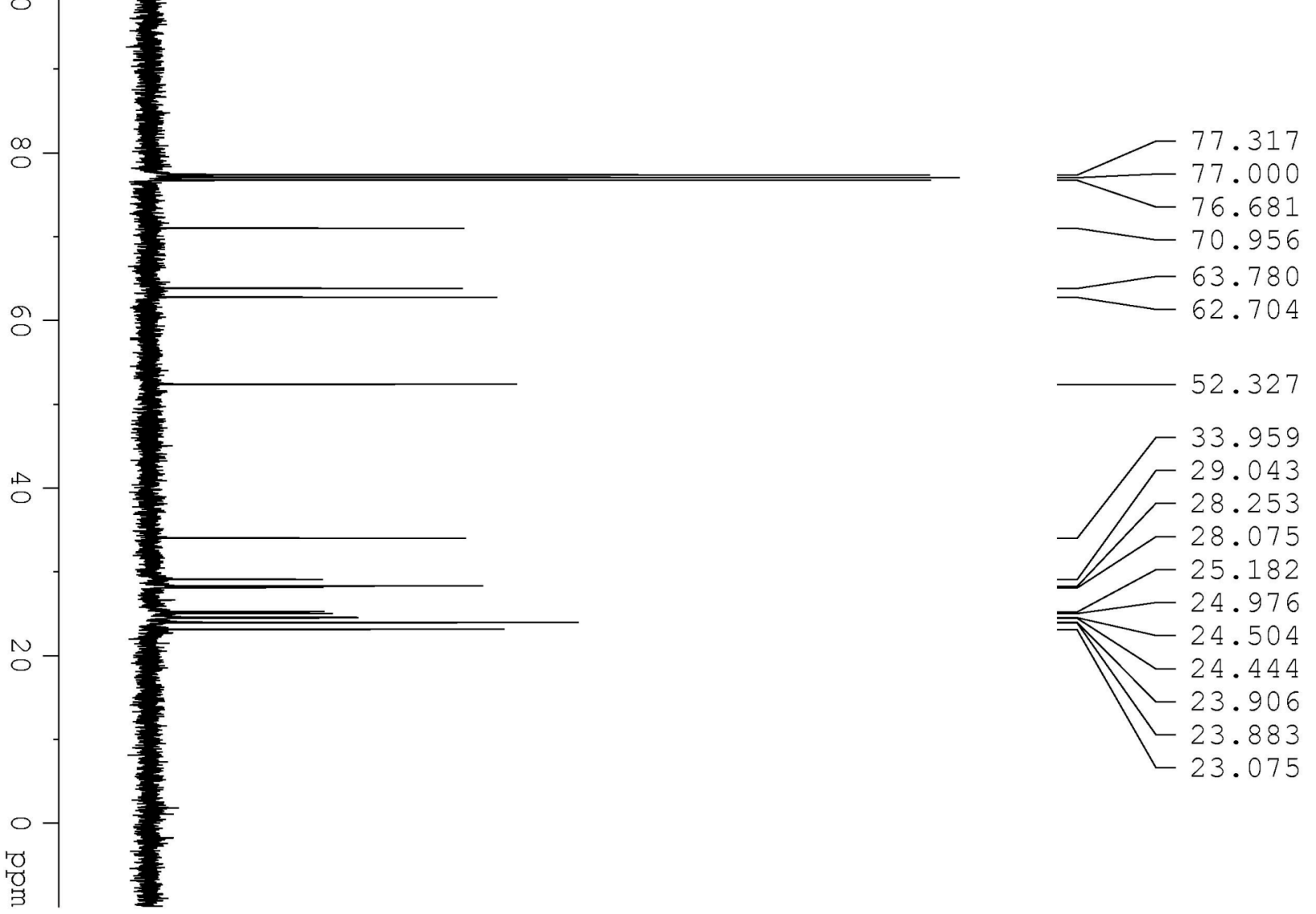


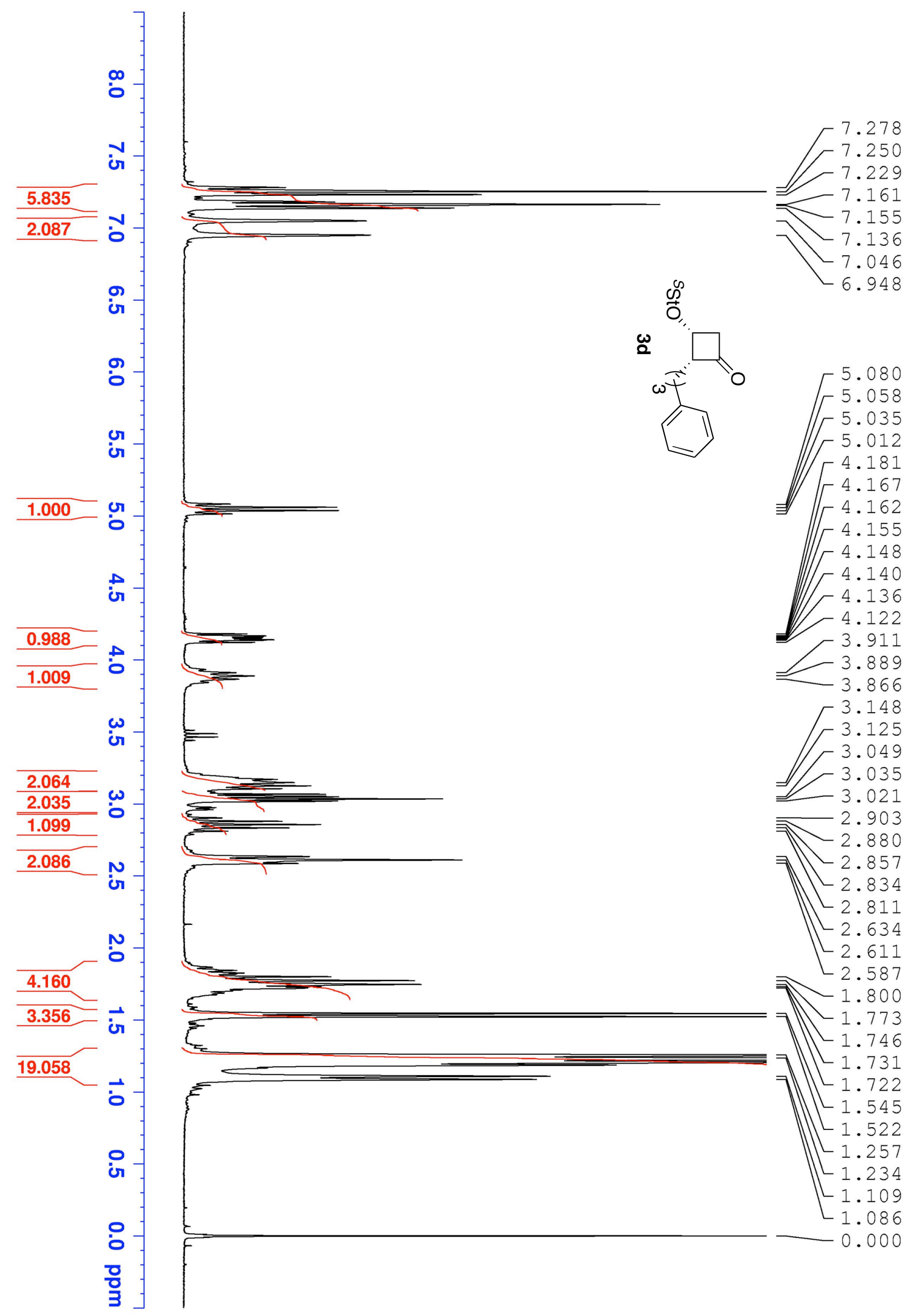




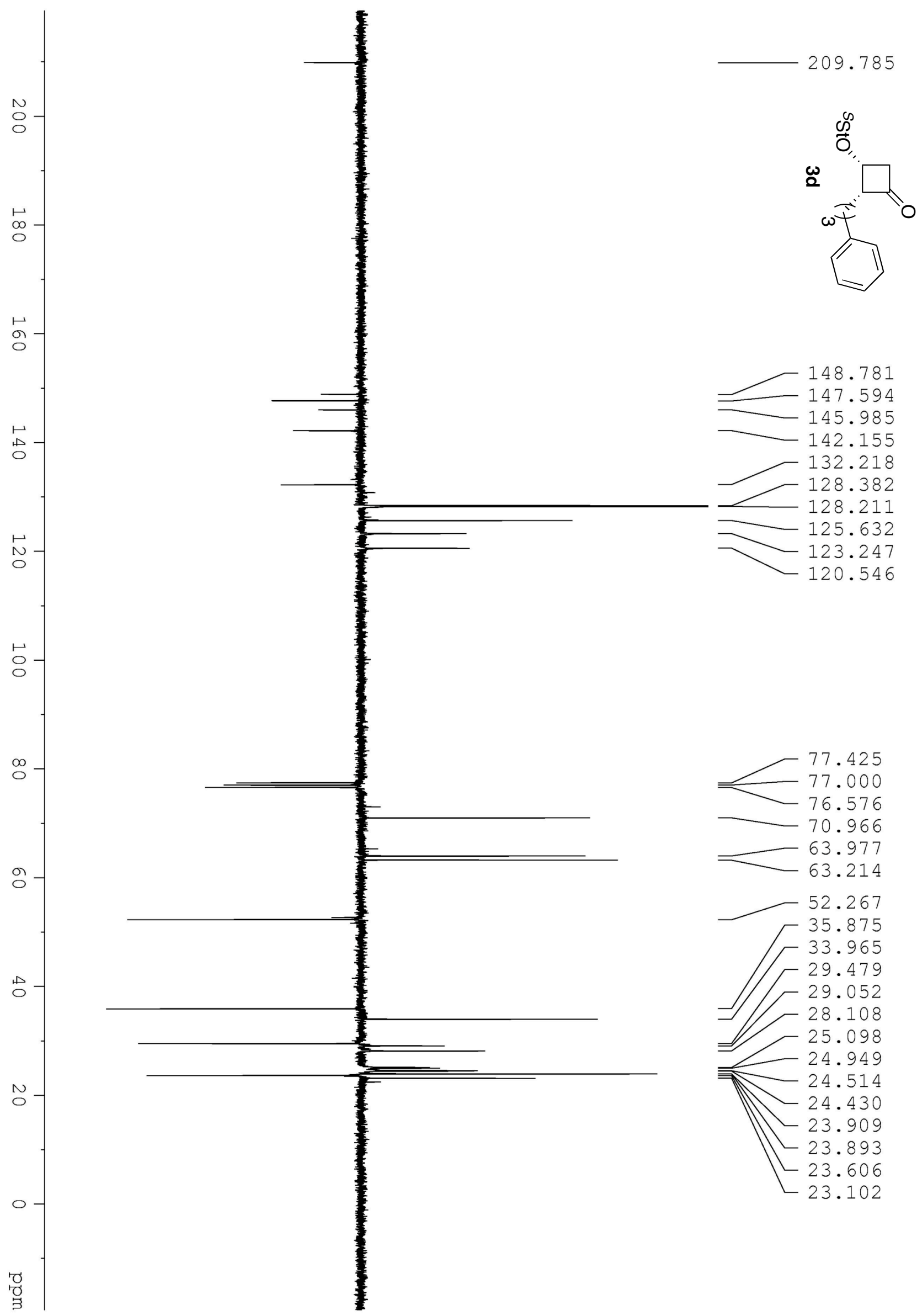




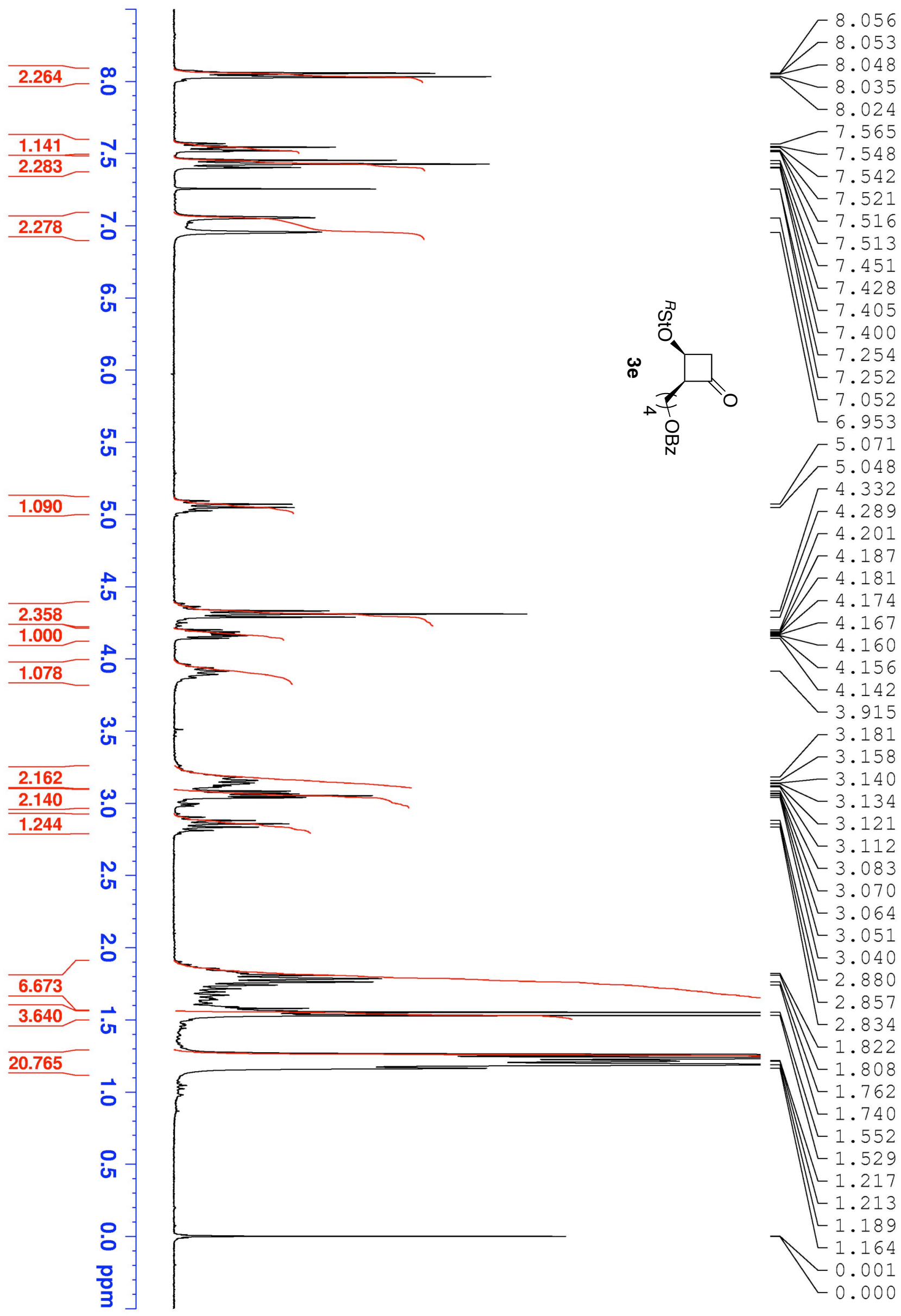




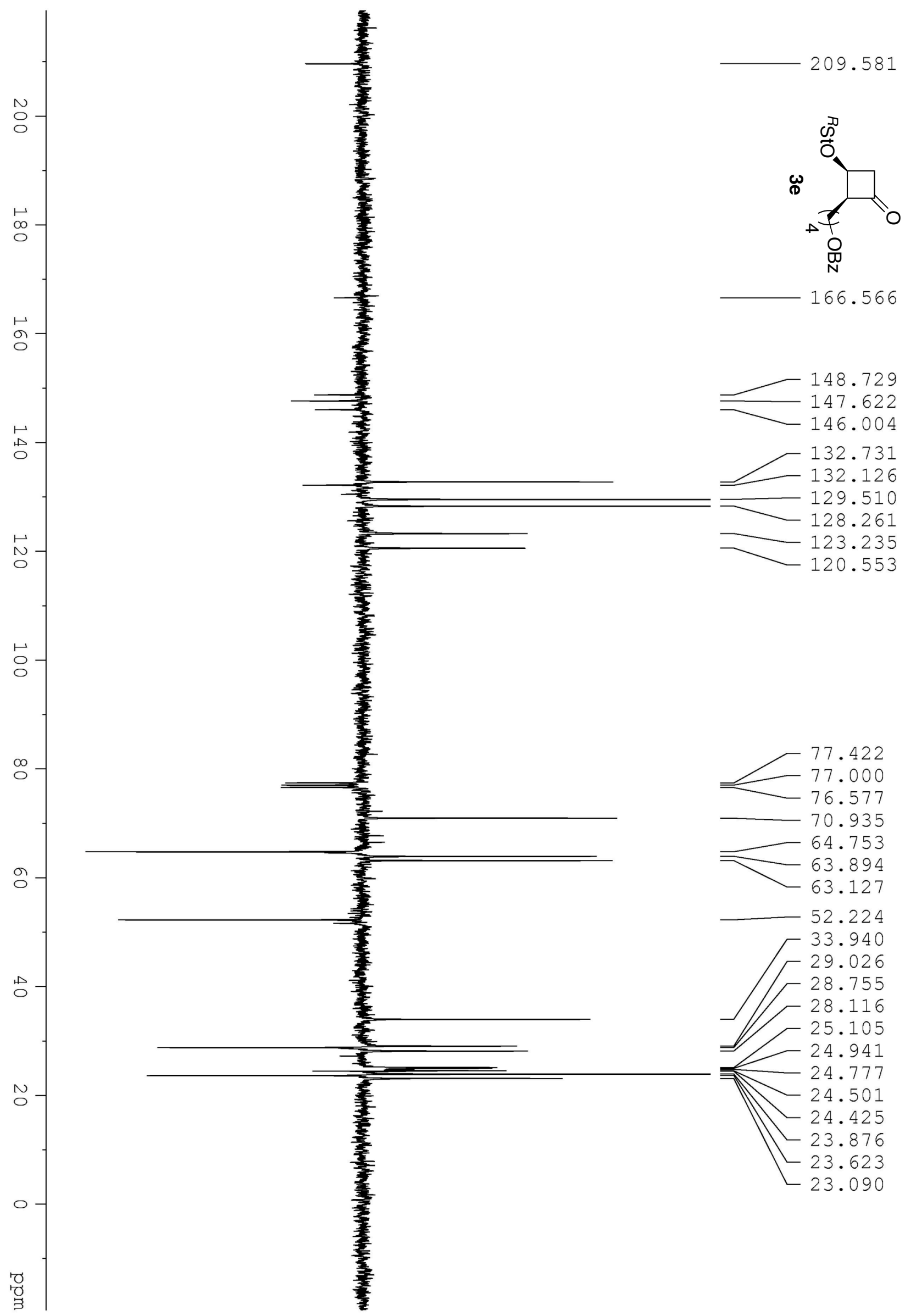




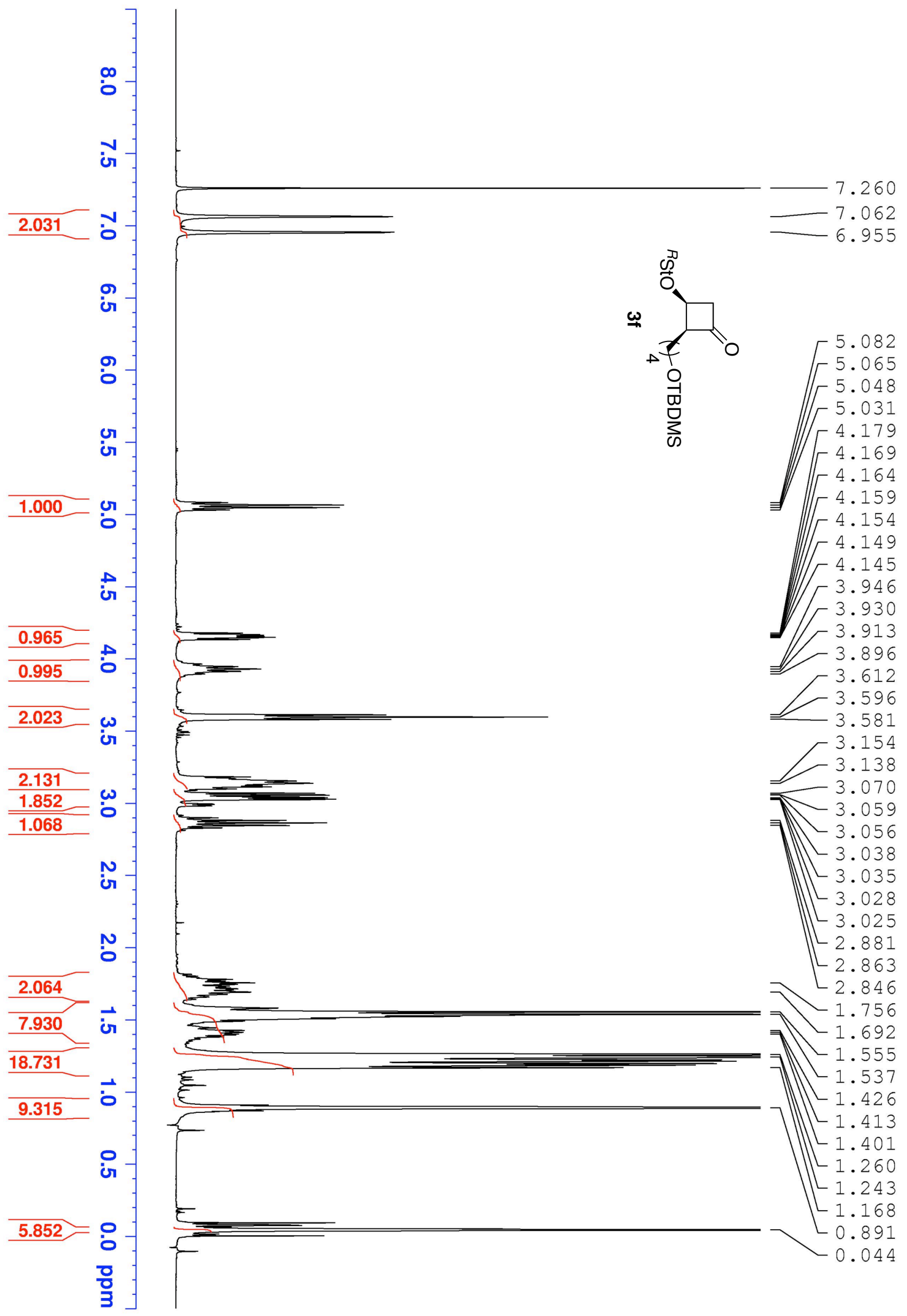




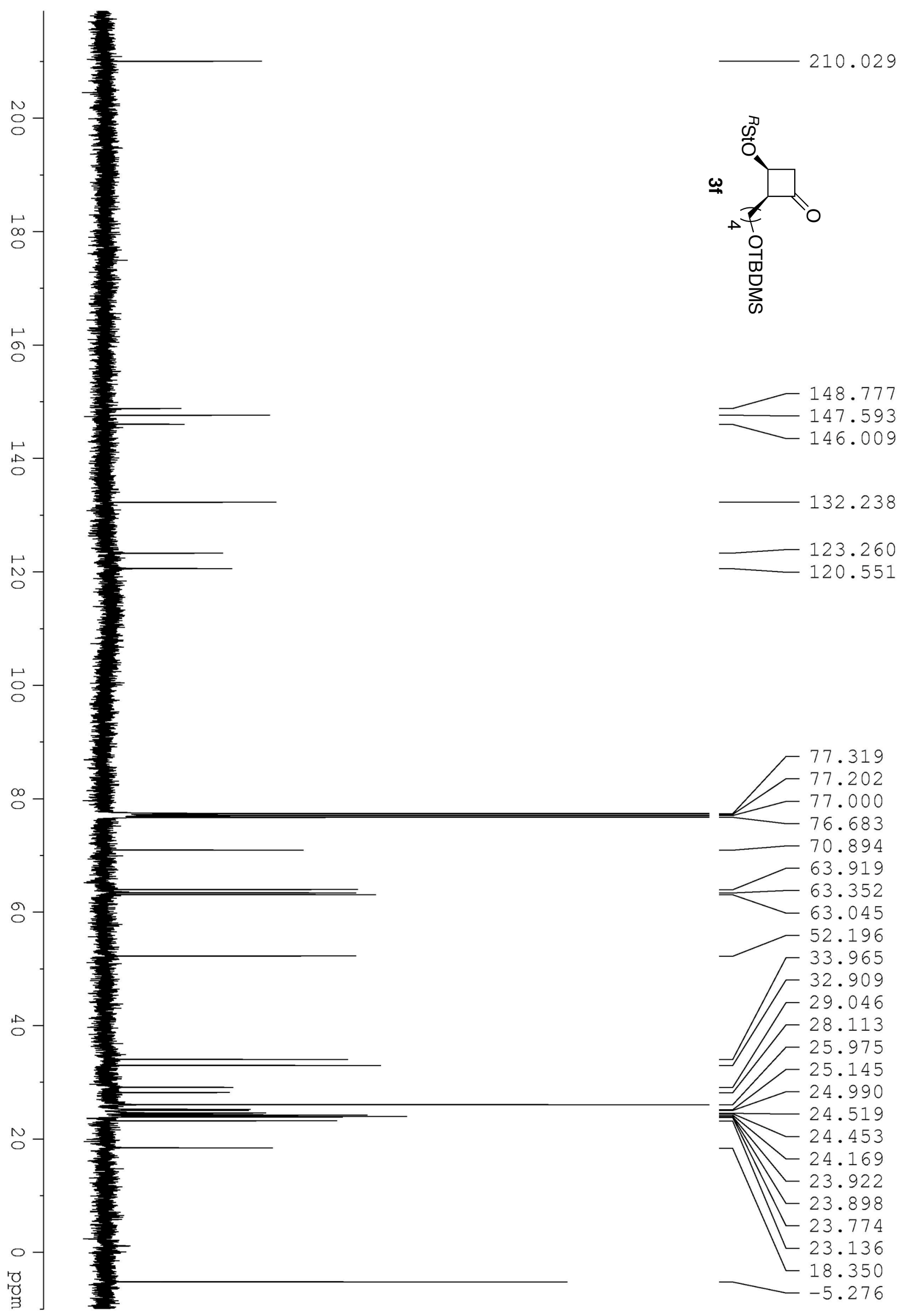




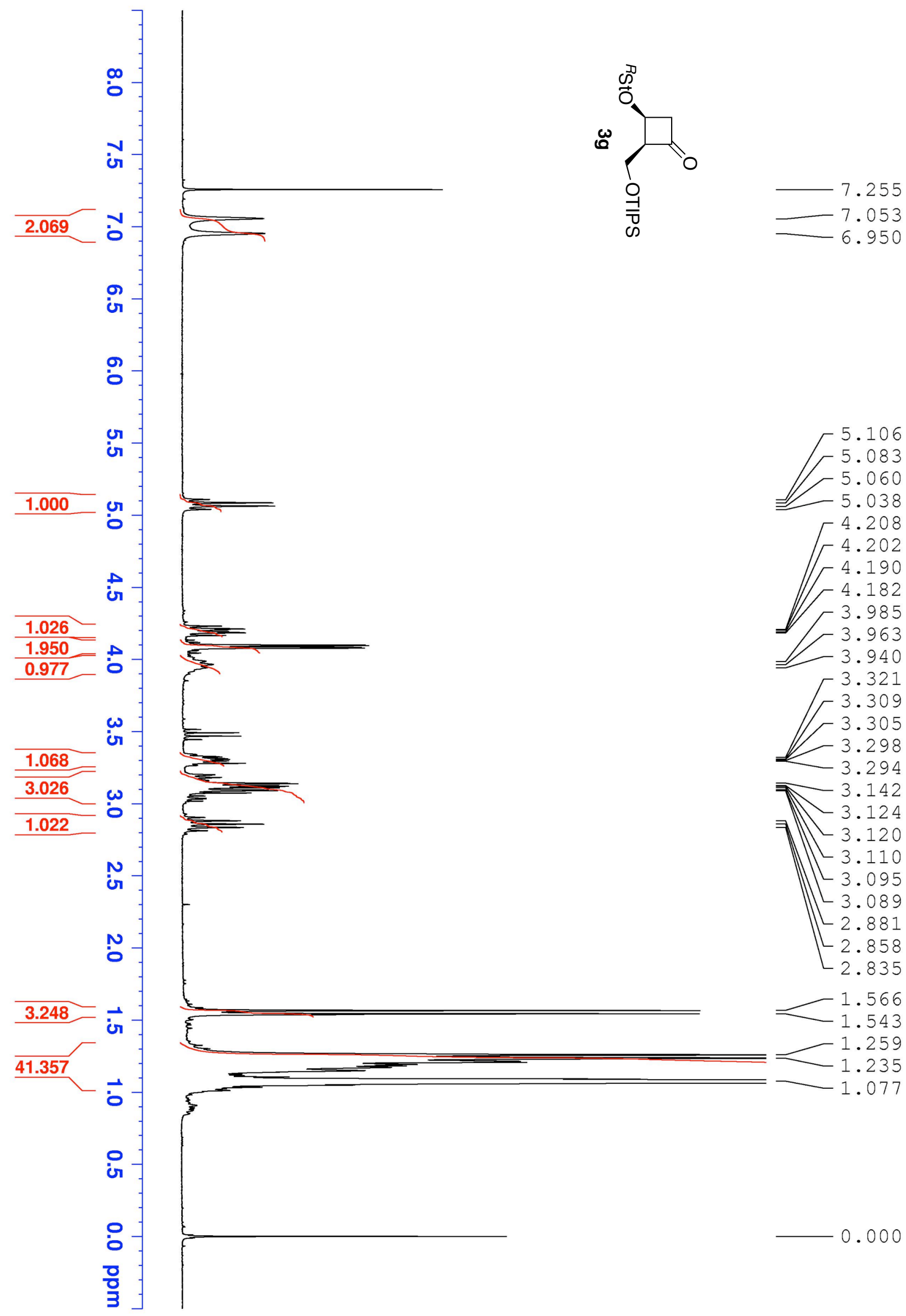




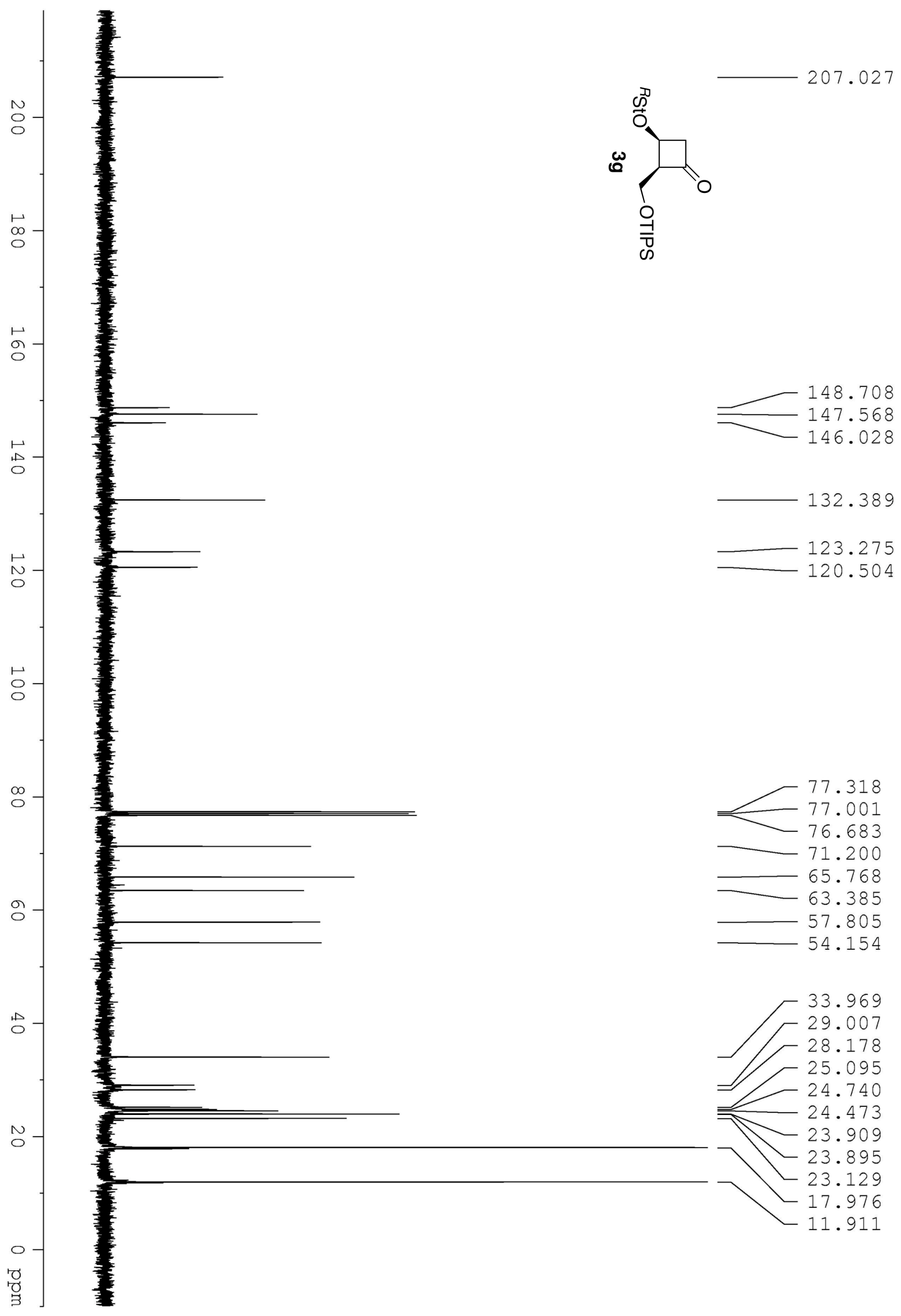




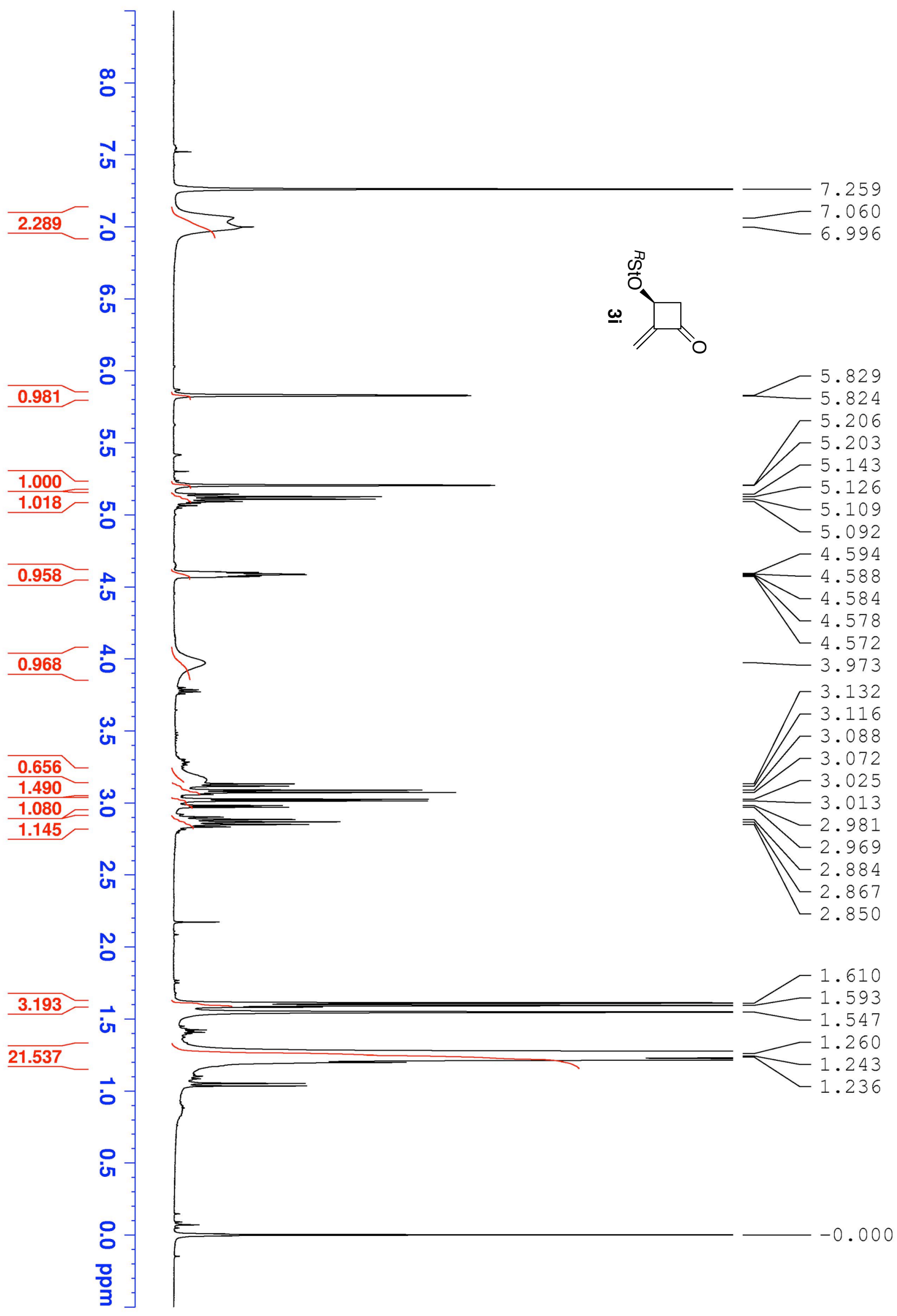




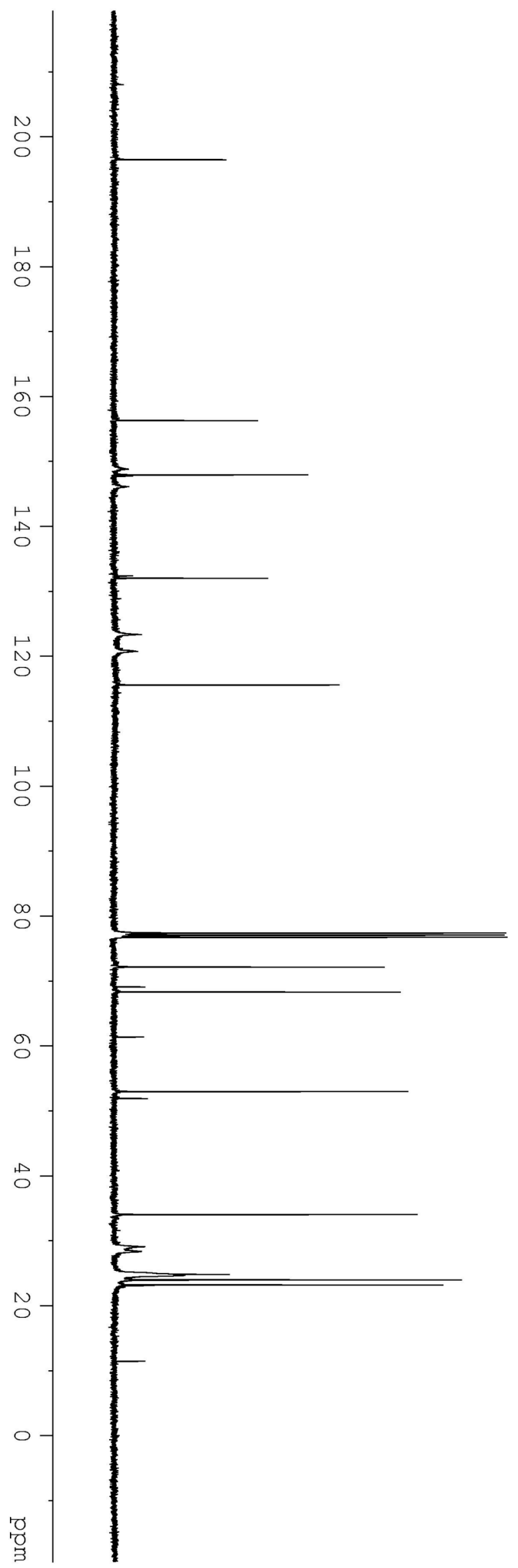

156.249

147.897
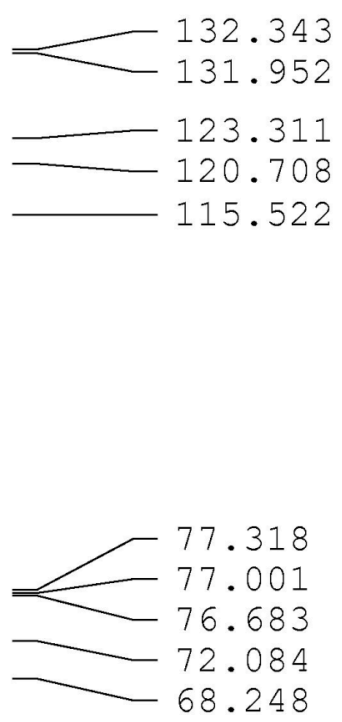

52.919

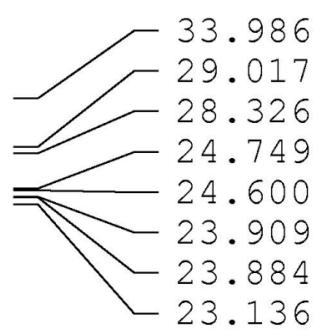




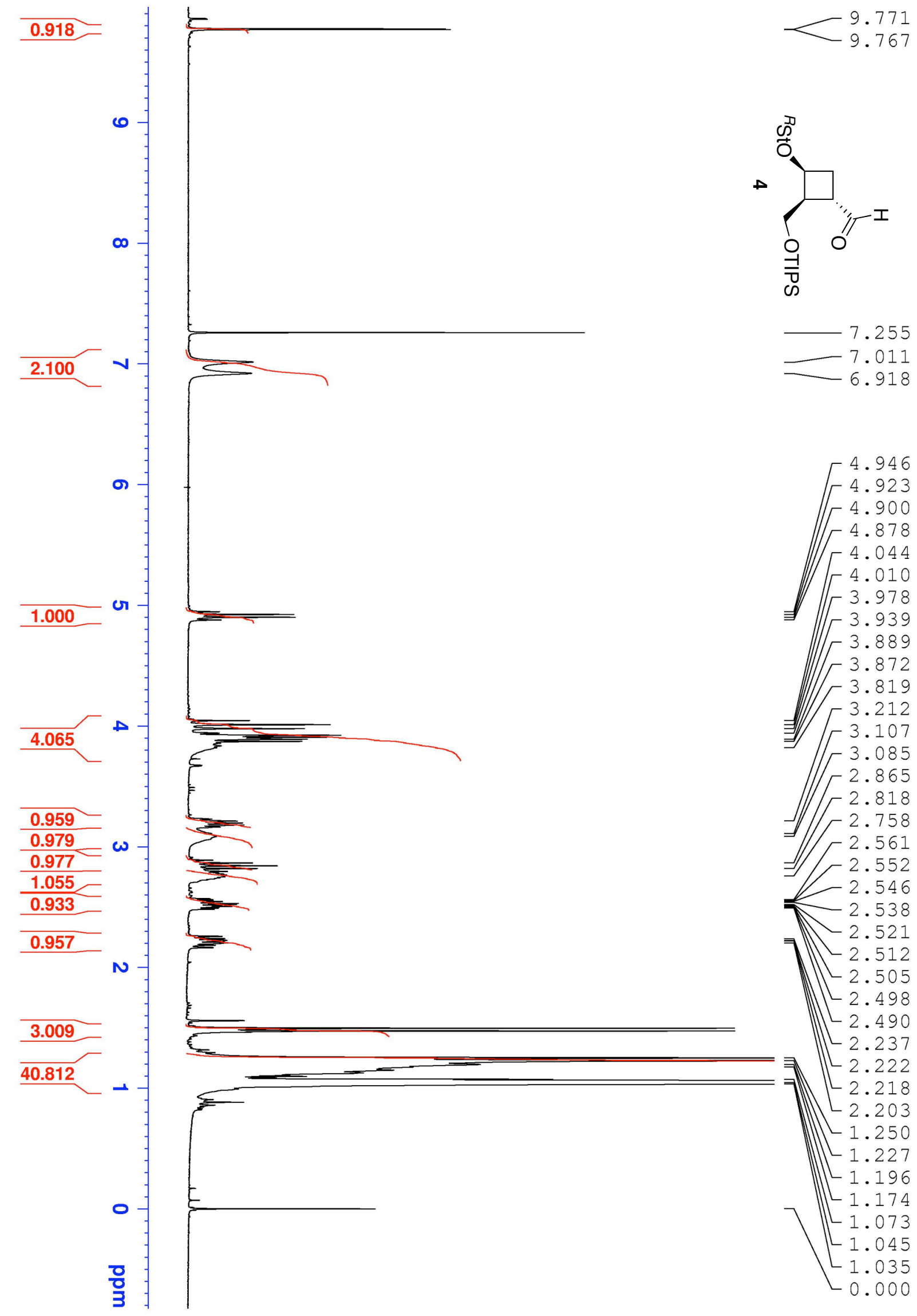




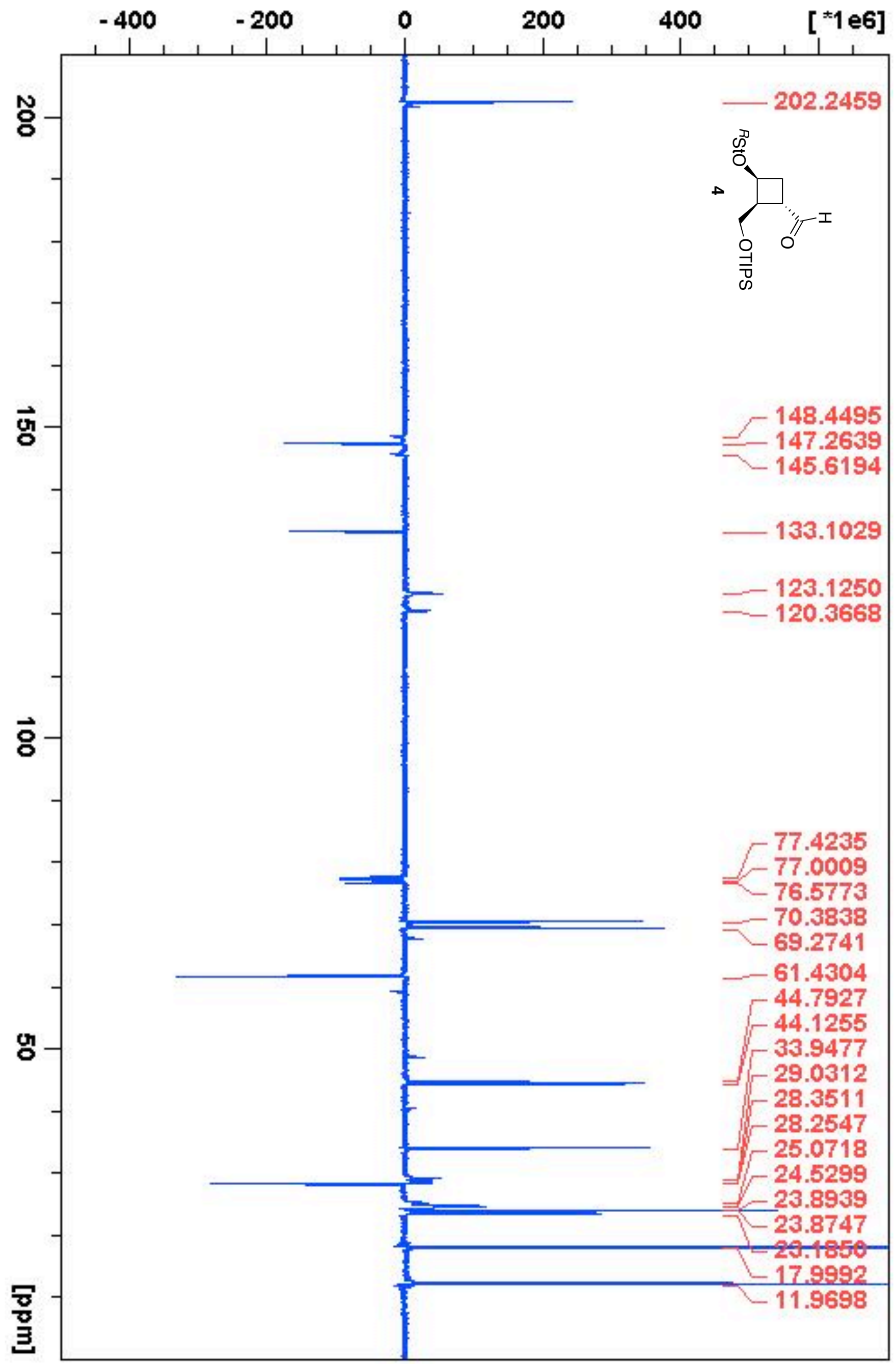




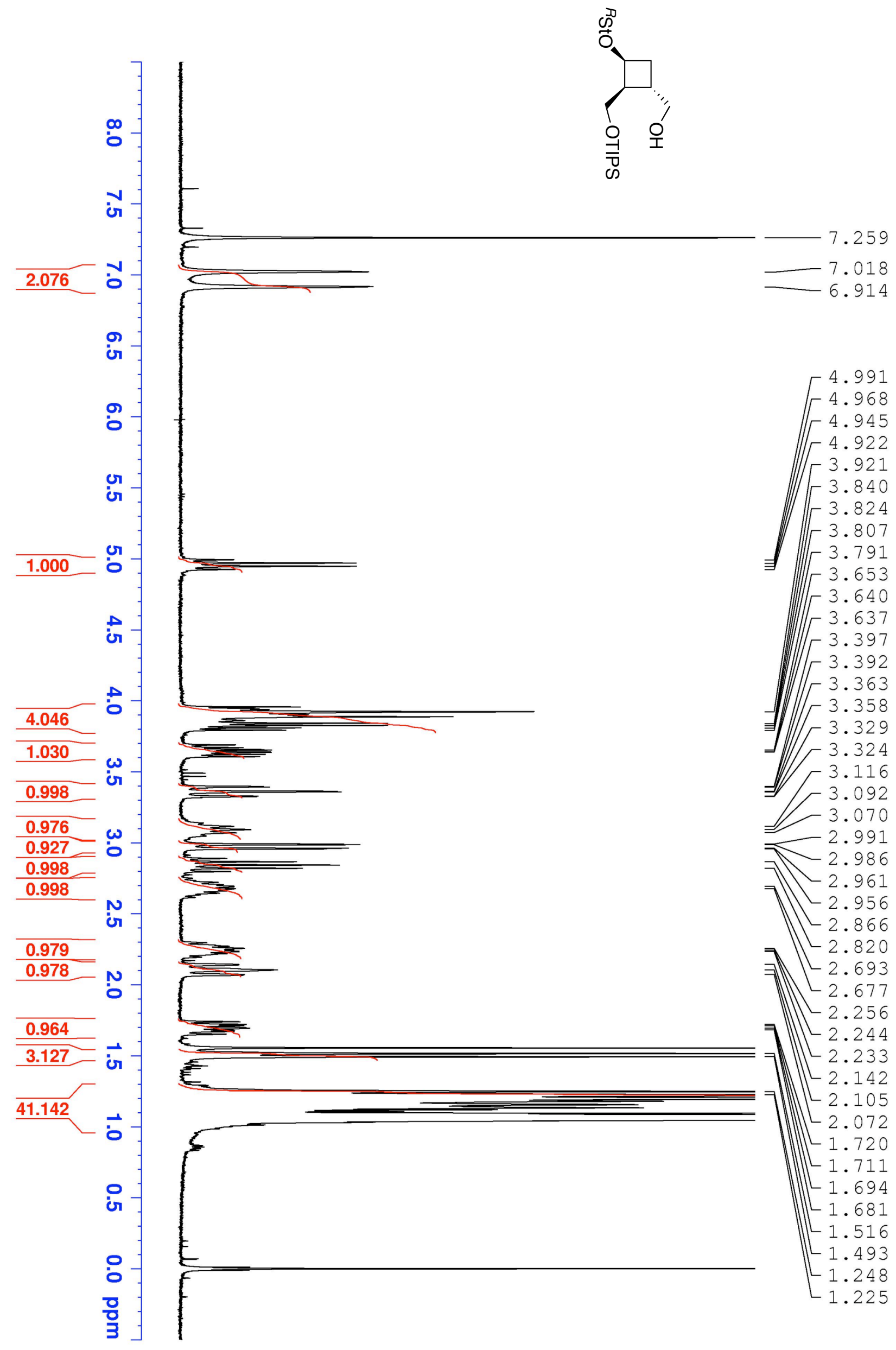




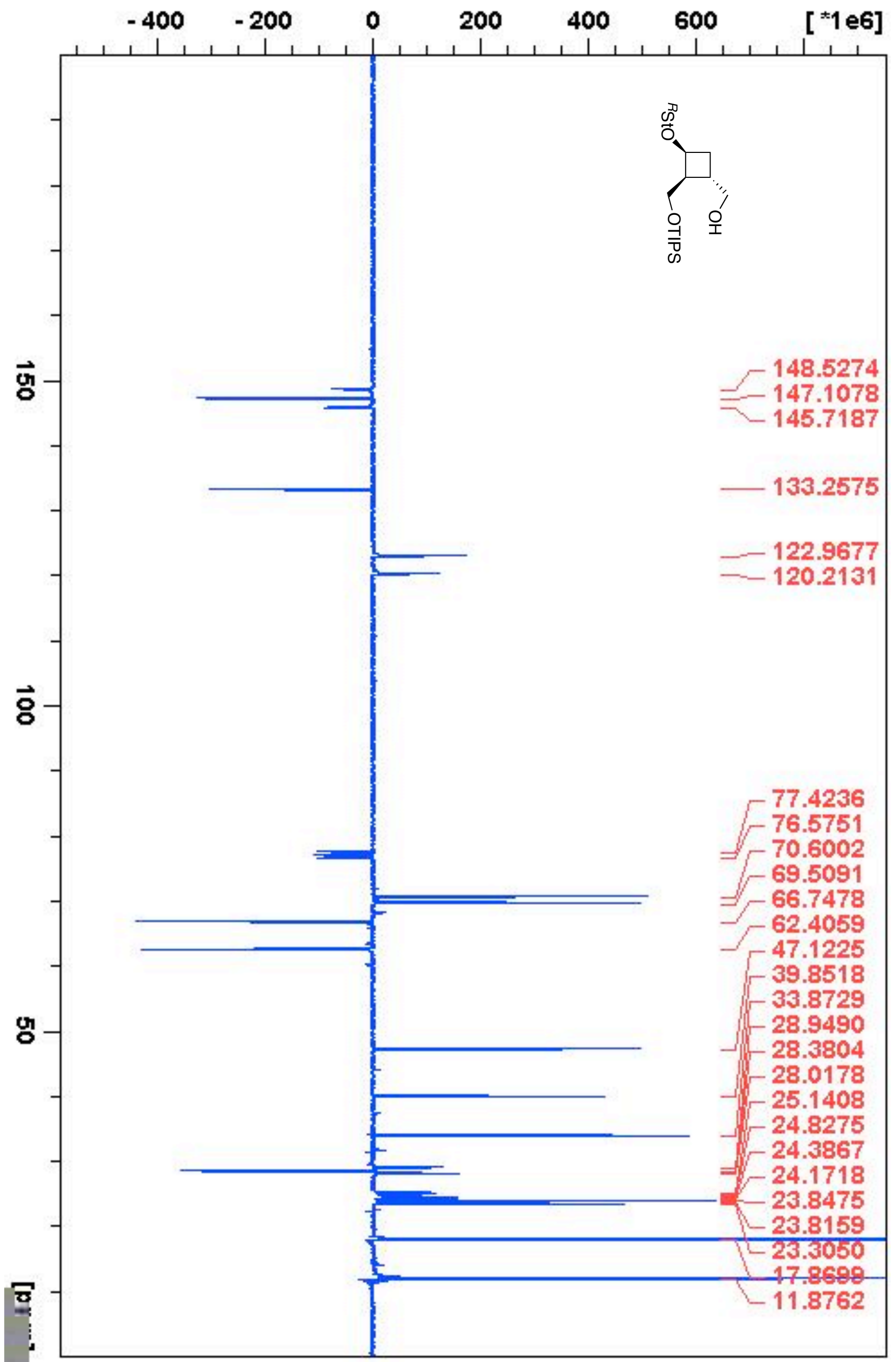




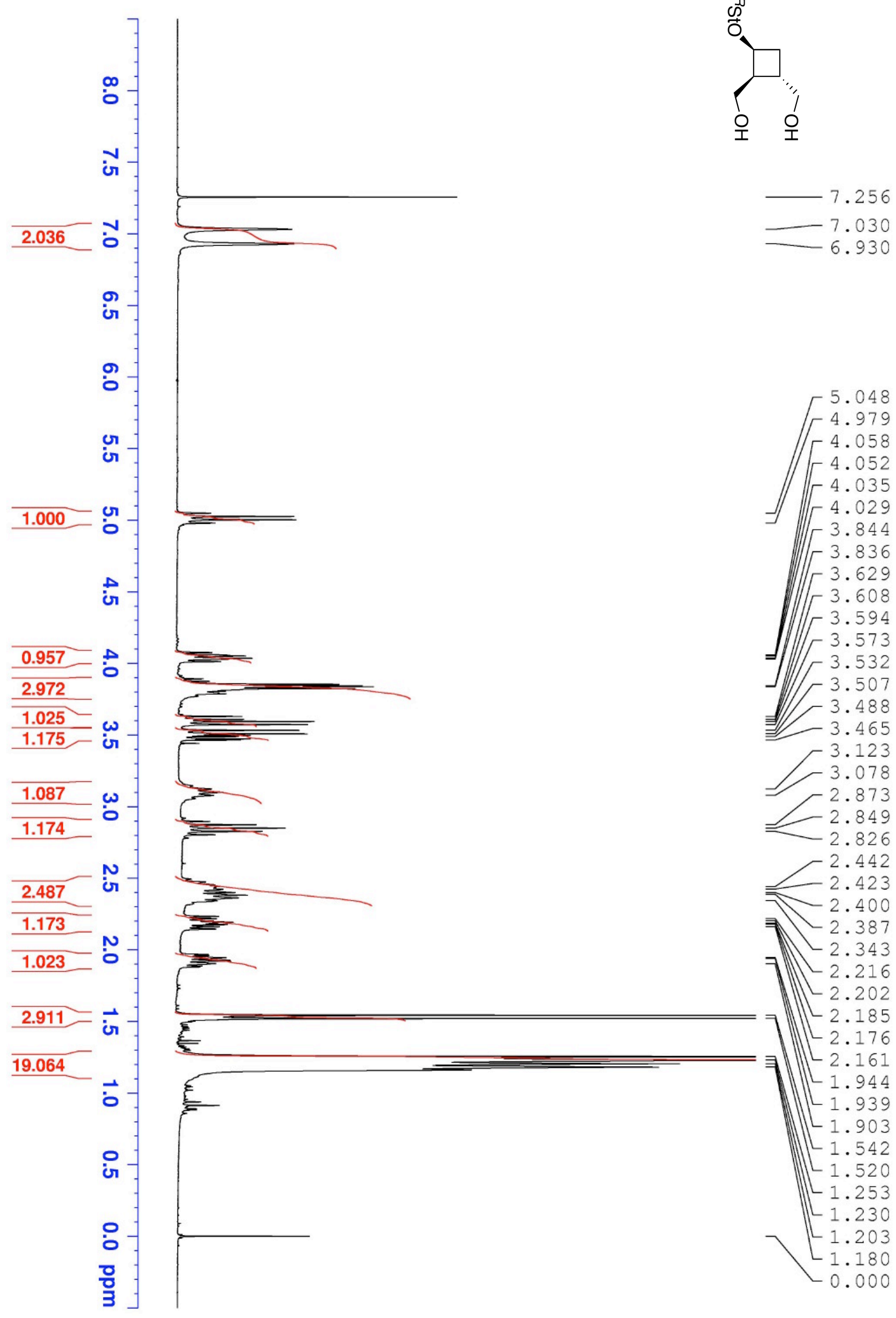




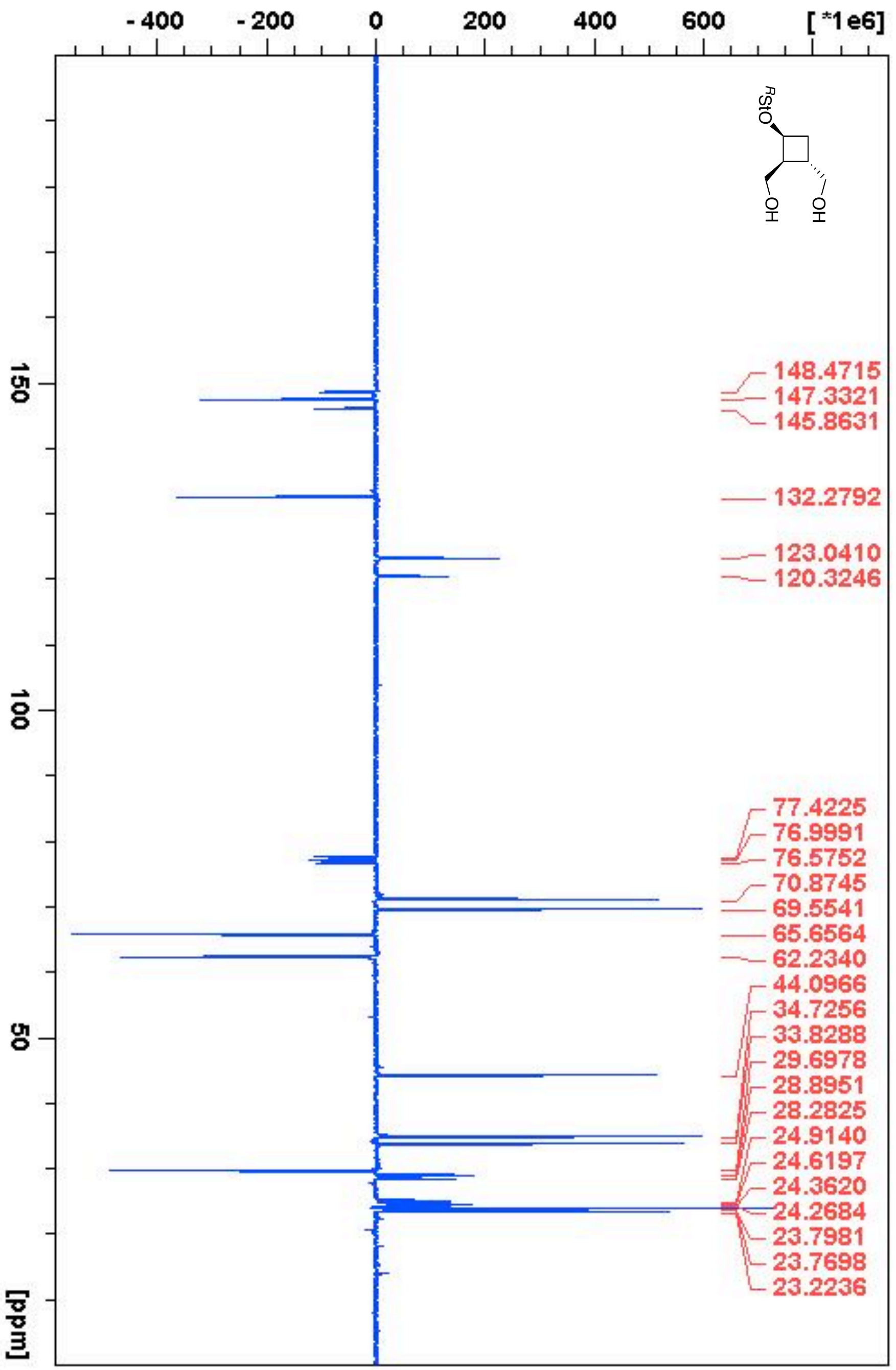




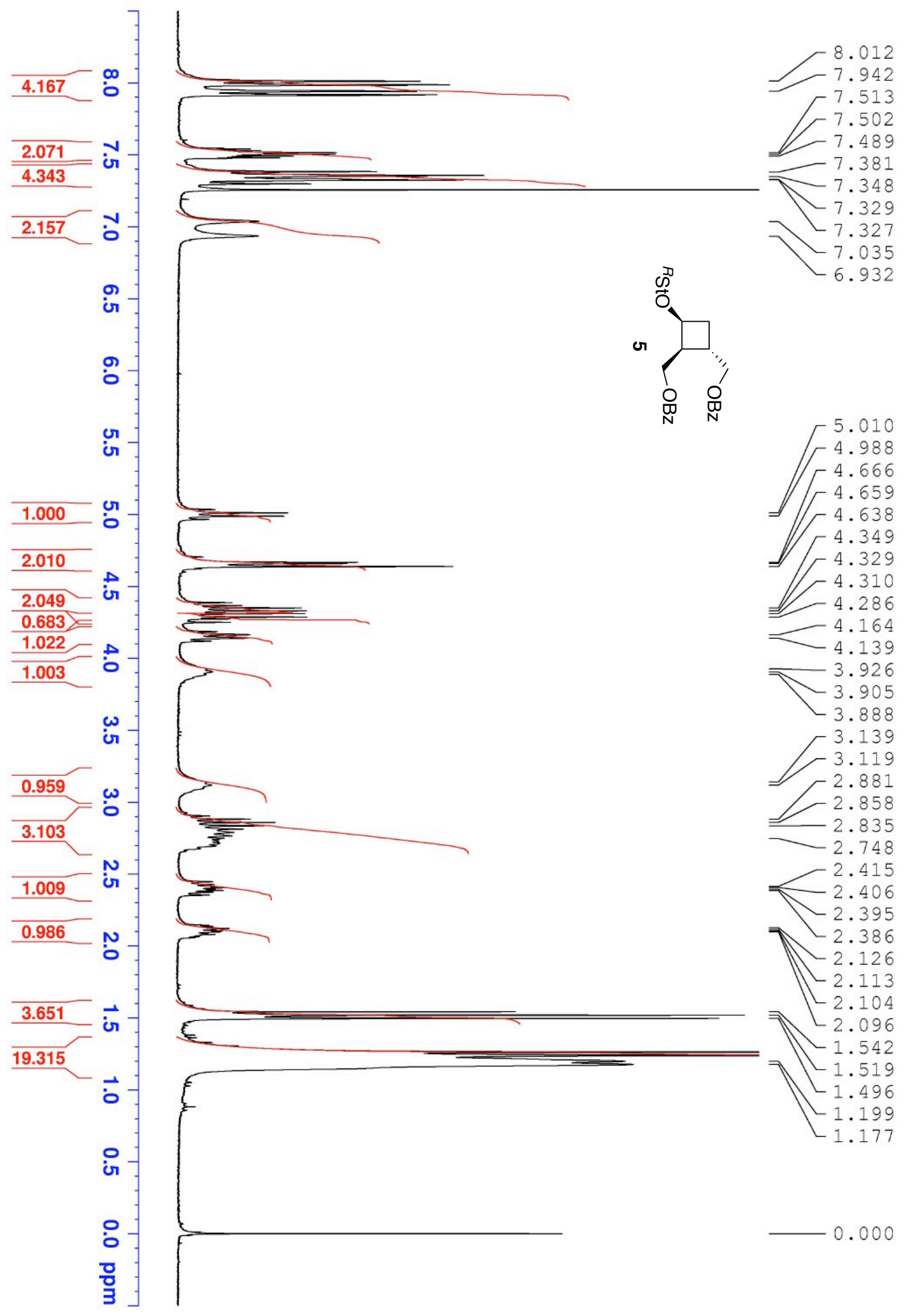




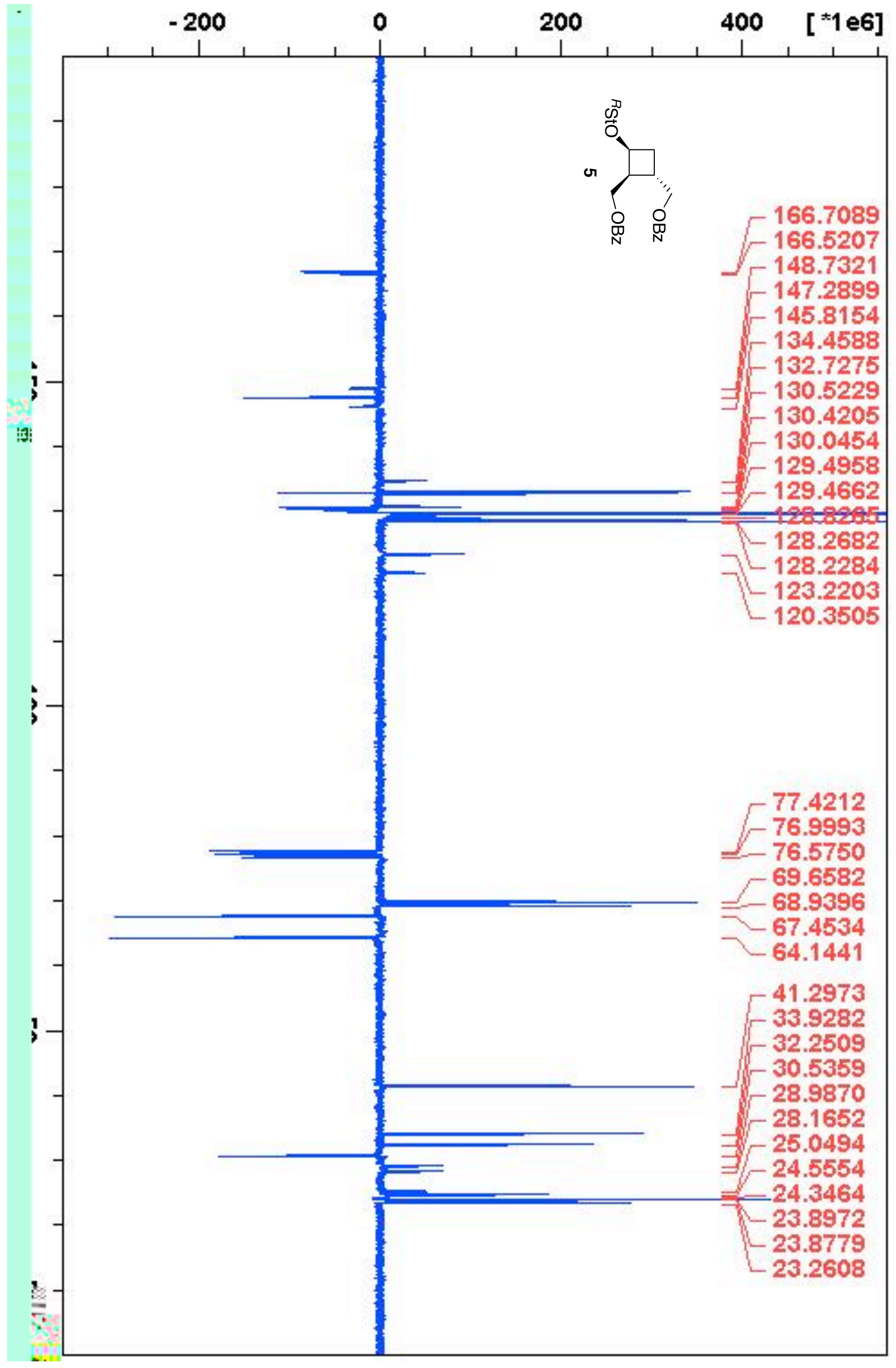




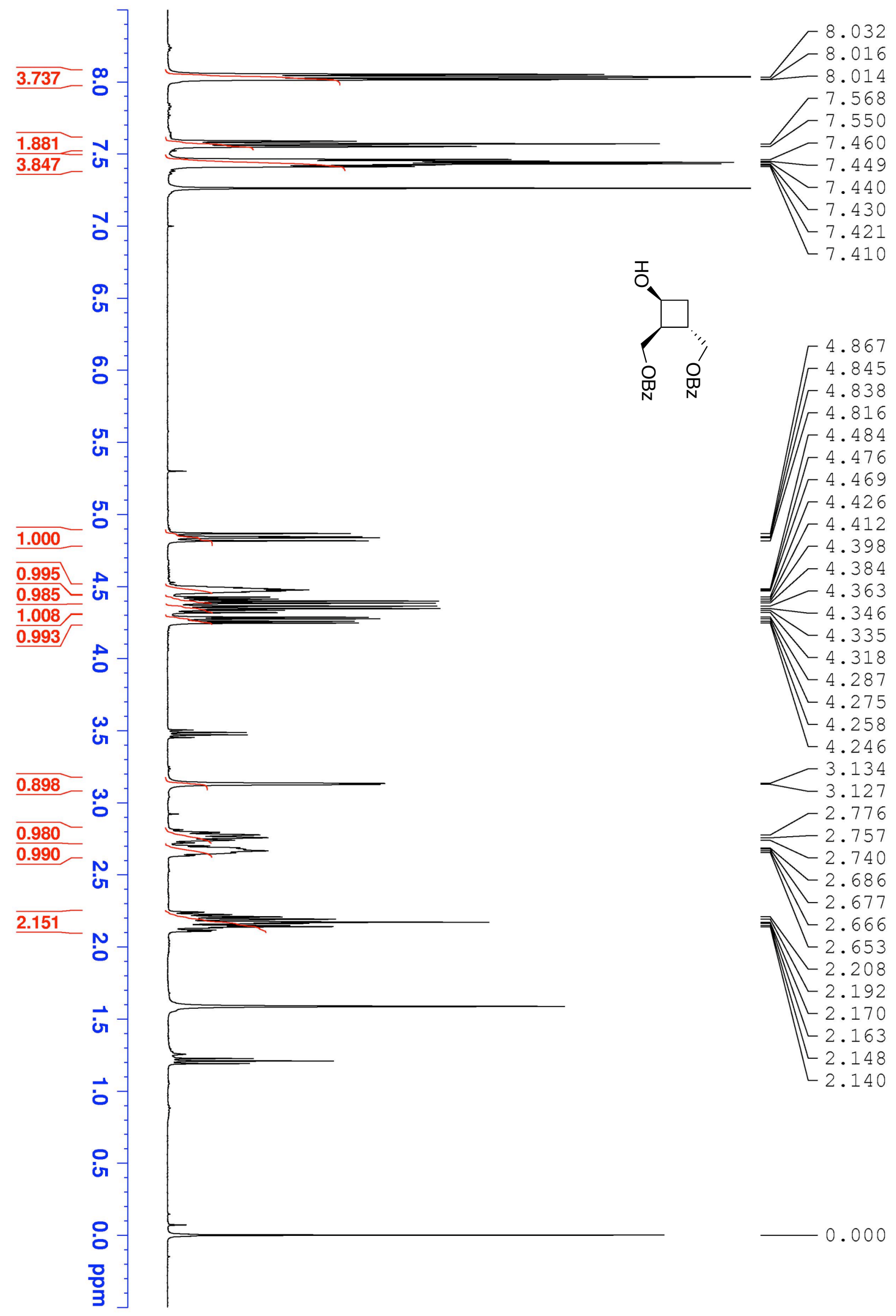




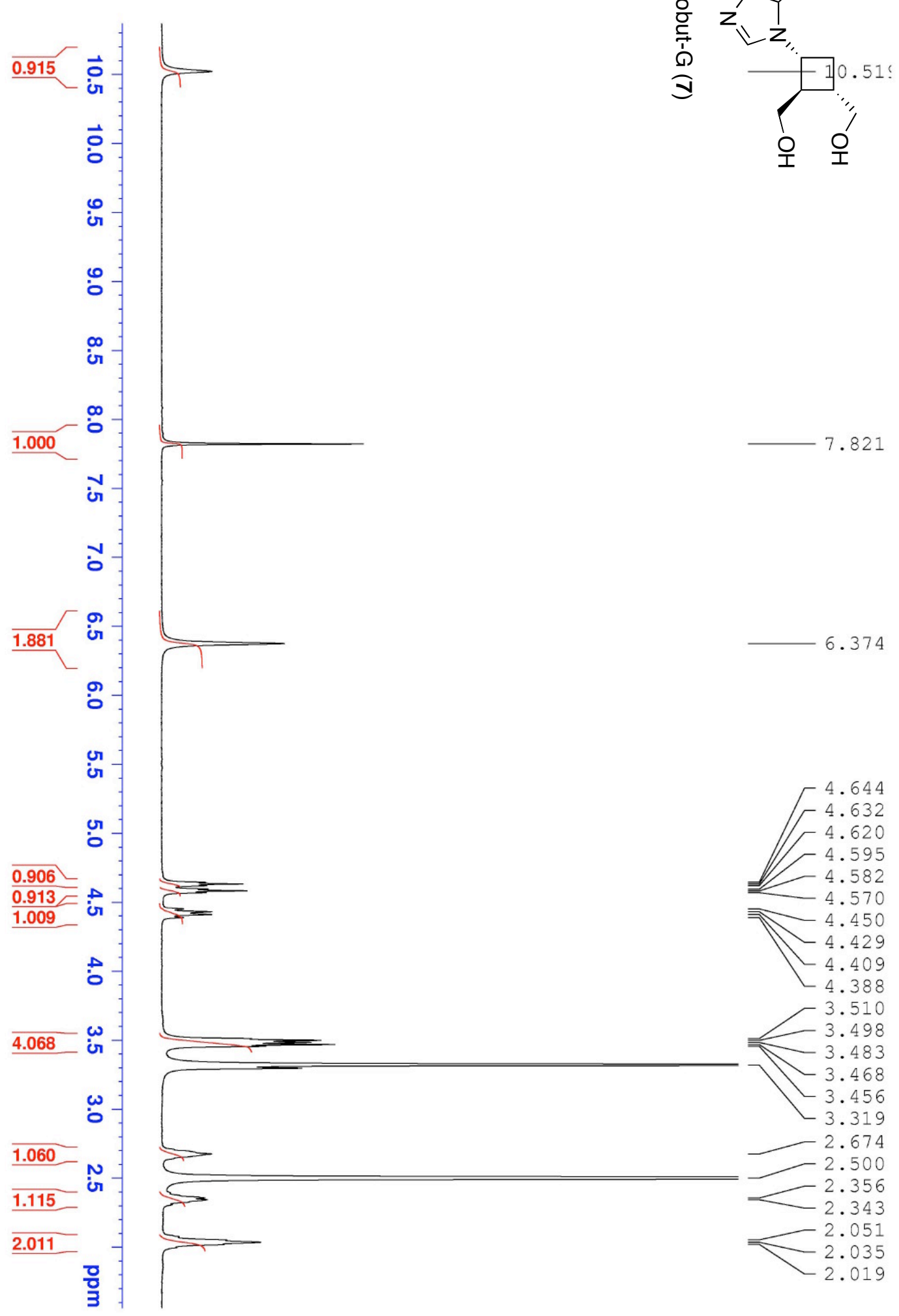




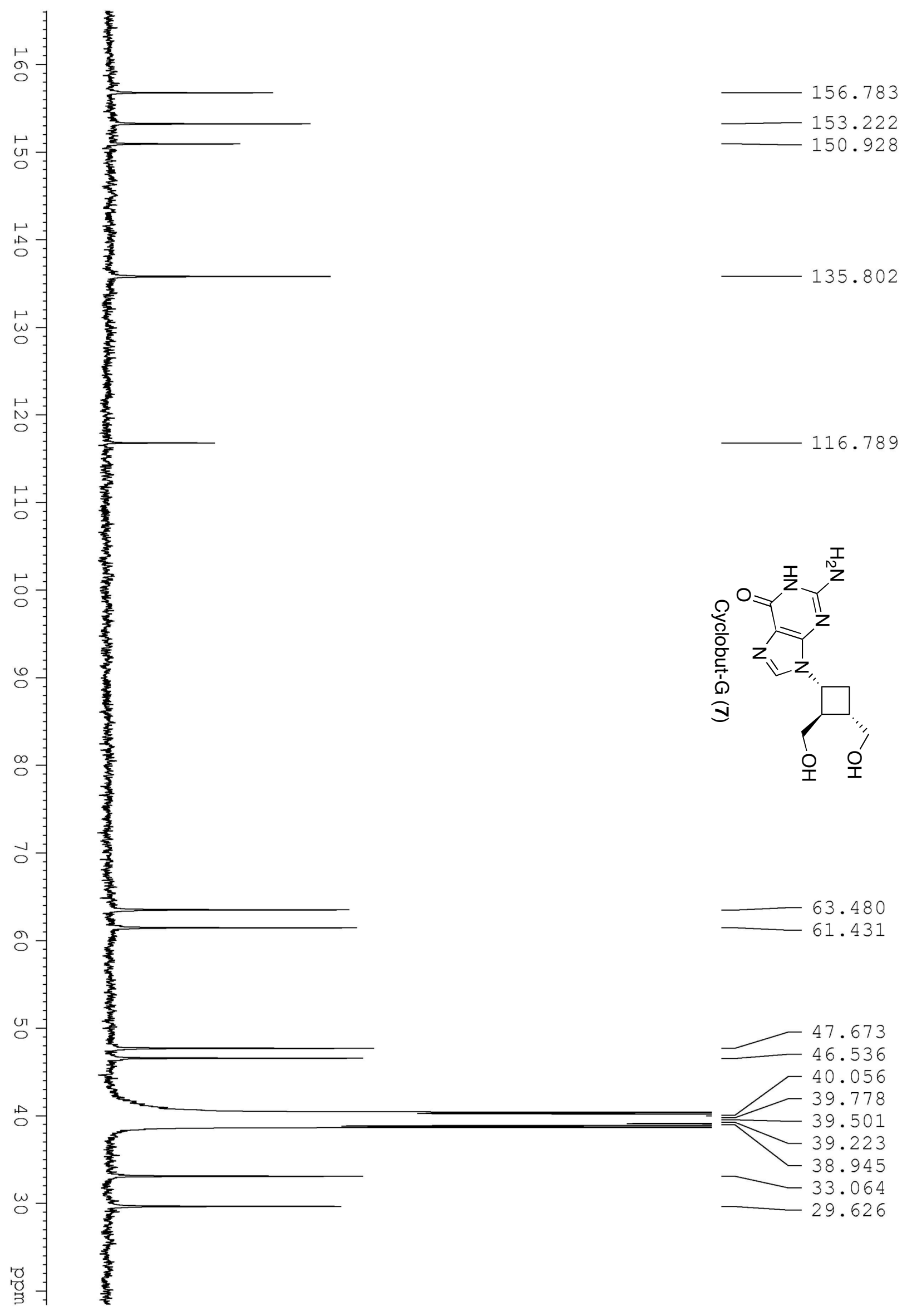

